\title{
Entropic fluctuations in Gaussian dynamical systems
}

\author{
V. JAKŠIĆ ${ }^{a}$, C.-A. PILLET ${ }^{b}$, A. SHIRIKYAN ${ }^{c}$ \\ ${ }^{a}$ Department of Mathematics and Statistics, McGill University \\ 805 Sherbrooke Street West \\ Montreal, QC, H3A 2K6, Canada \\ ${ }^{b}$ Aix-Marseille Université, CNRS UMR 7332, CPT, 13288 Marseille, France \\ Université de Toulon, CNRS UMR 7332, CPT, 83957 La Garde, France \\ ${ }^{c}$ Department of Mathematics, University of Cergy-Pontoise \\ CNRS UMR 8088, 2 avenue Adolphe Chauvin \\ 95302 Cergy-Pontoise, France
}

\begin{abstract}
We study non-equilibrium statistical mechanics of a Gaussian dynamical system and compute in closed form the large deviation functionals describing the fluctuations of the entropy production observable with respect to the reference state and the non-equilibrium steady state. The entropy production observable of this model is an unbounded function on the phase space, and its large deviation functionals have a surprisingly rich structure. We explore this structure in some detail.
\end{abstract}

\section{Contents}

1 Introduction 2

2 The model and results $\quad 3$

2.1 Gaussian dynamical systems . . . . . . . . . . . . . . . . . . 3

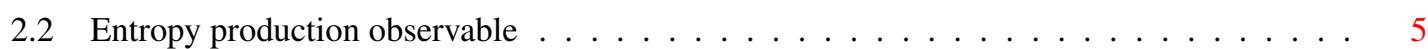

2.3 Non-equilibrium steady state . . . . . . . . . . . . . . . . . . . 6

2.4 Entropic fluctuations with respect to the reference state . . . . . . . . . . . . . 6

2.5 Entropic fluctuations with respect to the NESS . . . . . . . . . . . . . . . . 9

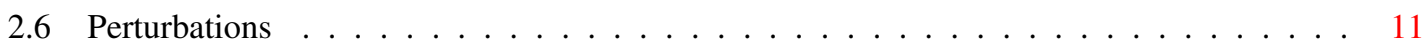

3 Examples 12 
3.1 Toy model . . . . . . . . . . . . . . . . . . . . . . . . . . . . 12

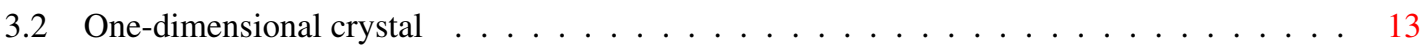

4 Proofs 16

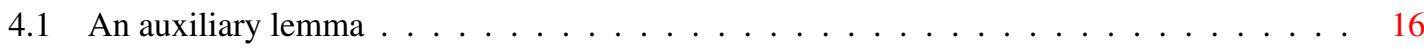

4.2 Proof of Proposition $2.1 \ldots \ldots \ldots \ldots \ldots \ldots$

4.3 Proof of Proposition $2.2 \ldots \ldots \ldots \ldots \ldots$

4.4 Proof of Proposition $2.3 \ldots \ldots \ldots \ldots \ldots \ldots$

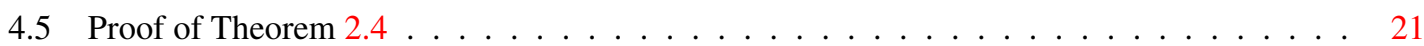

4.6 Proof of Theorem $2.5 \ldots \ldots \ldots \ldots \ldots \ldots \ldots \ldots$

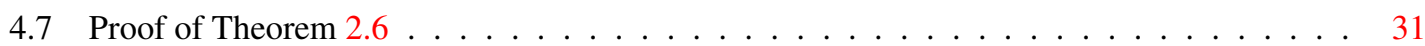

\section{Introduction}

In this paper, we prove and elaborate the results announced in Section 9 of [JPR]. We consider a dynamical system described by a real separable Hilbert space $\mathcal{K}$ and the equation of motion

$$
\frac{\mathrm{d}}{\mathrm{d} t} x_{t}=\mathcal{L} x_{t}, \quad x_{0} \in \mathcal{K},
$$

where $\mathcal{L}$ is a bounded linear operator on $\mathcal{K}$. Let $D$ be a strictly positive bounded symmetric operator on $\mathcal{K}$ and $\left(\mathfrak{X}, \omega_{D}\right)$ the Gaussian random field over $\mathcal{K}$ with zero mean value and covariance $D$. Eq. (1) induces a flow $\phi_{\mathcal{L}}=\left\{\phi_{\mathcal{L}}^{t}\right\}$ on $\mathfrak{X}$, and our starting point is the dynamical system $\left(\mathfrak{X}, \phi_{\mathcal{L}}, \omega_{D}\right)$ (its detailed construction is given in Section 2.1). We compute in closed form and under minimal regularity assumptions the nonequilibrium characteristics of this model by exploiting its Gaussian nature. In particular, we discuss the existence of a non-equilibrium steady state (NESS), compute the steady state entropy production, and study the large deviations of the entropy production observable w.r.t. both the reference state $\omega_{D}$ and the NESS. To emphasize the minimal mathematical structure behind the results, in the main body of the paper we have adopted an abstract axiomatic presentation. In Section 3, the results are illustrated on the example of the one-dimensional harmonic crystal. For additional information and a pedagogical introduction to the theory of entropic fluctuations in classical non-equilibrium statistical mechanics, we refer the reader to the reviews [RM, JPR].

There are very few models for which the large deviation functionals of the entropy production observable can be computed in a closed form, and we hope that our results may serve as a guide for future studies. In addition, an important characteristic of a Gaussian dynamical system is that its entropy production observable is an unbounded function on the phase space. This unboundedness has dramatic effects on the form and regularity properties of the large deviation functionals that require modifications of the celebrated fluctuation relations [ECM, ES, GC1, GC2]. Although this topic has received a considerable attention in the physics literature [BaCo, BGGZ, BJMS, Fa, HRS, Vi1, Vi2, ZC], to the best of our knowledge, it has not been studied in the mathematically rigorous literature on the subject. Thus, another goal of this paper is to initiate a research program dealing with mathematical theory of extended fluctuation relations in non-equilibrium statistical mechanics, which emerge when some of the usual regularity assumptions (such as compactness of the phase space, boundedness of the entropy production observable, smoothness of the time reversal map) are not satisfied.

The paper is organized as follows. In Section 2.1 we introduce Gaussian dynamical systems. In Section 2.2 we define the entropy production observable and describe its basic properties. In Section 2.3 we introduce the NESS. Our main results are stated in Sections 2.4 and 2.5. The entropy production observable is defined as the phase space contraction rate of the reference measure $\omega_{D}$ under the flow $\phi_{\mathcal{L}}$, and in Section 2.6 we examine the effects of a perturbation of the reference measure on the large deviation theory. In Section 3 
we illustrate our results on two classes of examples, toy models and harmonic chains. The proofs are given in Section 4.

The focus of this paper is the mathematics of the large deviation theory of the entropy production observable. The physical implications of our results will be discussed in the continuation of this paper [JPS].

Acknowledgment. This research was partly supported by CNRS PICS Project RESSPDE, NSERC (V.J.) and ANR (C.-A.P. and A.S.; grants 09-BLAN-0098 and 2011-BS01-015-01). The work of C.-A.P. has been carried out in the framework of the Labex Archimède (ANR-11-LABX-0033) and of the A*MIDEX project (ANR-11-IDEX-0001-02), funded by the "Investissements d'Avenir" French Government program managed by the French National Research Agency (ANR). C.-A.P. and A.S. also acknowledge the hospitality and excellent working conditions at the Mathematics Department of McGill University where part of this work was done. Another part of this work was done during the visits of V.J. to the Erwin Schrödinger Institute in Vienna and the Isaac Newton Institute in Cambridge. V.J. is grateful to these institutions for their hospitality.

\section{The model and results}

\subsection{Gaussian dynamical systems}

In order to setup our notation, we start with some basic facts about classical Gaussian dynamical systems. We refer the reader to [CFS] for a more detailed introduction to this subject.

Let $\Gamma$ be a countably infinite set and

$$
\mathfrak{X}=\left\{x=\left(x_{n}\right)_{n \in \Gamma} \mid x_{n} \in \mathbb{R}\right\}=\mathbb{R}^{\Gamma} .
$$

For $x \in \mathfrak{X}$ and $I \subset \Gamma$, we denote $x_{I}=\left(x_{i}\right)_{i \in I} \in \mathbb{R}^{I}$. Let $l=\left(l_{n}\right)_{n \in \Gamma}$ be a given sequence of strictly positive numbers such that $\sum_{n \in \Gamma} l_{n}=1$ (we shall call such a sequence a weight). Then

$$
d(x, y)=\sum_{n \in \Gamma} l_{n} \frac{\left|x_{n}-y_{n}\right|}{1+\left|x_{n}-y_{n}\right|}
$$

is a metric on $\mathfrak{X}$ and $(\mathfrak{X}, d)$ is a complete separable metric space. Its Borel $\sigma$-algebra $\mathcal{F}$ is generated by the set of all cylinders

$$
C_{I}(B)=\left\{x \in \mathfrak{X} \mid x_{I} \in B\right\},
$$

where $I \subset \Gamma$ is finite and $B \subset \mathbb{R}^{I}$ is a Borel set.

Let $\nu$ and $\omega$ be two Borel probability measures on $\mathfrak{X}$. We shall write $\nu \ll \omega$ when $\nu$ is absolutely continuous w.r.t. $\omega$. The corresponding Radon-Nikodym derivative is denoted by

$$
\Delta_{\nu \mid \omega}=\frac{\mathrm{d} \nu}{\mathrm{d} \omega} .
$$

We will also use the notation

$$
\ell_{\nu \mid \omega}=\log \Delta_{\nu \mid \omega} .
$$

The two measures $\nu$ and $\omega$ are called equivalent, denoted $\nu \simeq \omega$, if they are mutually absolutely continuous, i.e., $\omega \ll \nu$ and $\nu \ll \omega$. We adopt the shorthand $\nu(f)=\int_{\mathfrak{X}} f \mathrm{~d} \nu$. The relative entropy of $\nu$ w.r.t. $\omega$ is defined as

$$
\operatorname{Ent}(\nu \mid \omega)= \begin{cases}-\nu\left(\ell_{\nu \mid \omega}\right) & \text { if } \nu \ll \omega \\ -\infty & \text { otherwise. }\end{cases}
$$

\footnotetext{
${ }^{1}$ Throughout the paper we adopt the convention $\log x=-\infty$ for $x \leq 0$.
} 
We recall that $\operatorname{Ent}(\nu \mid \omega) \leq 0$, with equality iff $\nu=\omega$. For $\alpha \in \mathbb{R}$, the relative Rényi $\alpha$-entropy of $\nu$ w.r.t. $\omega$ is defined as

$$
\operatorname{Ent}_{\alpha}(\nu \mid \omega)= \begin{cases}\log \omega\left(\mathrm{e}^{\alpha \ell_{\nu \mid \omega}}\right) & \text { if } \nu \ll \omega, \\ -\infty & \text { otherwise }\end{cases}
$$

We denote by $\mathcal{K} \subset \mathfrak{X}$ the real Hilbert space with inner product

$$
(x, y)=\sum_{n \in \Gamma} x_{n} y_{n}
$$

$\left(\mathcal{K}=\ell_{\mathbb{R}}^{2}(\Gamma)\right)$, and by $\left\{\delta_{n}\right\}_{n \in \Gamma}$ its standard basis. The matrix elements of a linear operator $A$ on $\ell_{\mathbb{R}}^{2}(\Gamma)$ w.r.t. this basis are denoted by $A_{n m}=\left(\delta_{n}, A \delta_{m}\right)$.

Let $\mathfrak{X}_{l}, \mathfrak{X}_{l}^{*} \subset \mathfrak{X}$ be real Hilbert spaces with respective inner products

$$
(x, y)_{l}=\sum_{n \in \Gamma} l_{n} x_{n} y_{n}, \quad(x, y)_{l^{*}}=\sum_{n \in \Gamma} l_{n}^{-1} x_{n} y_{n},
$$

$\left(\mathfrak{X}_{l}^{*}\right.$ is the dual of $\mathfrak{X}_{l}$ w.r.t. the duality (3)). Clearly,

$$
\mathfrak{X}_{l}^{*} \subset \mathcal{K} \subset \mathfrak{X}_{l} \subset \mathfrak{X},
$$

with continuous and dense inclusions. All the measures on $(\mathfrak{X}, \mathcal{F})$ we will consider here will be concentrated on $\mathfrak{X}_{l}$.

Let $D$ be a bounded, strictly positive operator on $\mathcal{K}$. The centered Gaussian measure of covariance $D$ on $(\mathfrak{X}, \mathcal{F})$ is the unique Borel probability measure $\omega_{D}$ specified by its value on cylinders

$$
\omega_{D}\left(C_{I}(B)\right)=\frac{1}{\sqrt{\operatorname{det}\left(2 \pi D_{I}\right)}} \int_{B} \mathrm{e}^{-\frac{1}{2}\left(x, D_{I}^{-1} x\right)} \mathrm{d} x,
$$

where $D_{I}=\left[D_{i j}\right]_{i, j \in I}$. The measure $\omega_{D}$ is also uniquely specified by its characteristic function

$$
\mathfrak{X}_{l}^{*} \ni y \mapsto \chi(y)=\int_{\mathfrak{X}} \mathrm{e}^{\mathrm{i}(y, x)} \mathrm{d} \omega_{D}(x)=\mathrm{e}^{-(y, D y) / 2} .
$$

The bound

$$
\int_{\mathfrak{X}}\|x\|_{l}^{2} \mathrm{~d} \omega_{D}(x)=\int_{\mathfrak{X}} \sum_{n \in \Gamma} l_{n} x_{n}^{2} \mathrm{~d} \omega_{D}(x)=\sum_{n \in \Gamma} l_{n} D_{n n} \leq\|D\|,
$$

implies that $\omega_{D}\left(\mathfrak{X} \backslash \mathfrak{X}_{l}\right)=0$, i.e., that $\omega_{D}$ is concentrated on $\mathfrak{X}_{l}$.

Let $\mathcal{T}$ be the real vector space of all trace class operators on $\mathcal{K}$ and $\|T\|_{1}=\operatorname{tr}\left(\left(T^{*} T\right)^{1 / 2}\right)$ the trace norm on $\mathcal{T}$. The pair $\left(\mathcal{T},\|\cdot\|_{1}\right)$ is a real Banach space. By the Feldman-Hajek-Shale theorem, two Gaussian measures $\omega_{D_{1}}$ and $\omega_{D_{2}}$ on $(\mathfrak{X}, \mathcal{F})$ are equivalent iff $T=D_{2}^{-1}-D_{1}^{-1} \in \mathcal{T}$. In this case, one has

$$
\begin{aligned}
\Delta_{\omega_{D_{2}} \mid \omega_{D_{1}}}(x) & =\sqrt{\operatorname{det}\left(I+D_{1} T\right)} \mathrm{e}^{-(x, T x) / 2}, \\
\operatorname{Ent}\left(\omega_{D_{2}} \mid \omega_{D_{1}}\right) & =\frac{1}{2} \operatorname{tr}\left(D_{1} T\left(I+D_{1} T\right)^{-1}\right)-\frac{1}{2} \log \operatorname{det}\left(I+D_{1} T\right) .
\end{aligned}
$$

Note that $\operatorname{det}\left(I+D_{1} T\right)=\operatorname{det}\left(I+D_{1}^{1 / 2} T D_{1}^{1 / 2}\right)=\operatorname{det}\left(D_{1}^{1 / 2} D_{2}^{-1} D_{1}^{1 / 2}\right)>0$.

Let $\mathcal{L}$ be a bounded linear operator on $\mathcal{K}$ such that $\mathcal{L}^{*} \mathfrak{X}_{l}^{*} \subset \mathfrak{X}_{l}^{*}$. It follows that $\mathcal{L}$ has a continuous extension to $\mathfrak{X}_{l}$ which we also denote by $\mathcal{L}$. For $x \in \mathfrak{X}$ and $t \in \mathbb{R}$ we set

$$
\phi_{\mathcal{L}}^{t}(x)= \begin{cases}\mathrm{e}^{t \mathcal{L}} x & \text { if } x \in \mathfrak{X}_{l}, \\ x & \text { if } x \notin \mathfrak{X}_{l} .\end{cases}
$$

The map $(t, x) \mapsto \phi_{\mathcal{L}}^{t}(x)$ is measurable and $\phi_{\mathcal{L}}=\left\{\phi_{\mathcal{L}}^{t}\right\}_{t \in \mathbb{R}}$ is a group of automorphisms of the measurable space $(\mathfrak{X}, \mathcal{F})$ describing the time evolution. We shall call $\phi_{\mathcal{L}}$ the dynamics generated by $\mathcal{L}$ and $\left(\mathfrak{X}, \phi_{\mathcal{L}}, \omega_{D}\right)$ a Gaussian dynamical system. Note that for $\omega_{D}$-almost all $x \in \mathfrak{X}, \phi_{\mathcal{L}}^{t}(x)=\mathrm{e}^{t \mathcal{L}} x$ for all $t \in \mathbb{R}$. 


\subsection{Entropy production observable}

Our starting point is the dynamical system $(\mathfrak{X}, \phi, \omega)$, where $\phi$ is the dynamics on $\mathfrak{X}$ generated by $\mathcal{L}$ and $\omega$ is the centered Gaussian measure with covariance $D$ (from now on, $\mathcal{L}$ and $D$ are fixed, and we shall omit explicit reference to them). The measure $\omega$ is sometimes called the initial or the reference state of the system. Observables are measurable functions $f: \mathfrak{X} \rightarrow \mathbb{C}$. They evolve according to

$$
f_{t}(x)=f \circ \phi^{t}(x) \text {. }
$$

The expectation of an observable $f$ at time $t \in \mathbb{R}$ is given by

$$
\omega_{t}(f)=\omega\left(f_{t}\right)=\int f_{t}(x) \mathrm{d} \omega(x)
$$

where $\omega_{t}=\omega \circ \phi^{-t}$ is the centered Gaussian measure on $(\mathfrak{X}, \mathcal{F})$ with covariance

$$
D_{t}=\mathrm{e}^{t \mathcal{L}} D \mathrm{e}^{t \mathcal{L}^{*}}
$$

$D_{t}$ is a bounded strictly positive operator on $\ell_{\mathbb{R}}^{2}(\Gamma)$ and $\omega_{t}\left(\mathfrak{X}_{l}\right)=1$ for all $t$. By the Feldman-Hajek-Shale theorem, the two measures $\omega_{t}$ and $\omega$ are equivalent iff $T_{t}:=D_{t}^{-1}-D^{-1} \in \mathcal{T}$. We shall assume more:

(G1) The map $\mathbb{R} \ni t \mapsto T_{t} \in \mathcal{T}$ is differentiable at $t=0$.

As will be seen later, this condition implies that the function $t \mapsto T_{t}$ is differentiable for all $t$. The entropy production observable (or phase space contraction rate) for $(\mathfrak{X}, \phi, \omega)$ is defined by

$$
\sigma(x)=\left.\frac{\mathrm{d}}{\mathrm{d} t} \ell_{\omega_{t} \mid \omega}(x)\right|_{t=0}, \quad x \in \mathcal{K} .
$$

A simple computation shows that (cf. (37))

$$
\sigma(x)=(x, \varsigma x)-\operatorname{tr}(D \varsigma),
$$

where

$$
\varsigma=-\left.\frac{1}{2} \frac{\mathrm{d} T_{t}}{\mathrm{~d} t}\right|_{t=0}
$$

and the derivative is understood in the sense of $\mathcal{T}$ (in particular, $\varsigma \in \mathcal{T}$ ). Since $\mathcal{T}$ is continuously embedded in the Banach space of all bounded operators on $\mathcal{K}$, we have

$$
\varsigma=\frac{1}{2}\left(\mathcal{L}^{*} D^{-1}+D^{-1} \mathcal{L}\right)
$$

Remark. If $A$ is a self-adjoint element of $\mathcal{T}$, then the quadratic form $(x, A x)$ has a unique extension from $\mathcal{K}$ to an element of $L^{1}(\mathfrak{X}, \mathrm{d} \omega)$. With a slight abuse of notation, we shall also denote this extension by $(x, A x)$ (see Lemma 4.1 below for a more precise statement). Thus, the entropy production observable (7) is a continuous function on $\mathcal{K}$ and an integrable function on $\mathfrak{X}$ w.r.t. the measure $\omega$.

Proposition 2.1 Suppose that (G1) holds. Then:

(1) The function $\mathbb{R} \ni t \mapsto \sigma_{t} \in L^{1}(\mathfrak{X}, \mathrm{d} \omega)$ is continuous.

(2) $\ell_{\omega_{t} \mid \omega}=\int_{0}^{t} \sigma_{-s} \mathrm{~d}$ s holds as the Riemann integral of a continuous $L^{1}(\mathfrak{X}, \mathrm{d} \omega)$-valued function. It also holds for $\omega$-almost every $x \in \mathfrak{X}$ as the Lebesgue integral of a real-valued function.

(3) The function $\mathbb{R} \ni t \mapsto \mathrm{e}^{\ell_{\omega_{t} \mid \omega}} \in L^{1}(\mathfrak{X}, \mathrm{d} \omega)$ is $C^{1}$ and

$$
\frac{\mathrm{d}}{\mathrm{d} t} \mathrm{e}^{\ell_{\omega_{t} \mid \omega}}=\mathrm{e}^{\ell_{\omega_{t} \mid \omega}} \sigma_{-t} .
$$


(4) $\omega_{t}(\sigma)=\operatorname{tr}\left(\varsigma\left(D_{t}-D\right)\right)$ and in particular $\omega(\sigma)=0$.

(5) $\operatorname{Ent}\left(\omega_{t} \mid \omega\right)=-\int_{0}^{t} \omega_{s}(\sigma) \mathrm{d} s$.

In specific examples, it may happen that only finitely many matrix elements $\varsigma_{n m}$ are non-zero, and in this case the map $x \mapsto \sigma(x)$ is continuous on $\mathfrak{X}$. The function $\sigma$ is bounded only in the trivial case $\sigma=0$. Note that $\sigma=0$ iff $\omega_{t}=\omega$ for all $t$; this follows, for instance, from the cocycle property (38).

\subsection{Non-equilibrium steady state}

Our next assumptions are:

(G2) There are some numbers $0<m<M<\infty$ such that $m \leq D_{t} \leq M$ for all $t \in \mathbb{R}$.

(G3) The following strong limits exist:

$$
\underset{t \rightarrow \pm \infty}{\mathrm{S}-\lim _{t} D_{t}=D_{ \pm}}
$$

It is clear that $m \leq D_{ \pm} \leq M$, and $\mathcal{L} D_{ \pm}+D_{ \pm} \mathcal{L}^{*}=0$. In what follows, we set

$$
\delta=\frac{m}{M-m} .
$$

Let $\omega_{ \pm}$be the centered Gaussian measure on $(\mathfrak{X}, \mathcal{F})$ with covariance $D_{ \pm}$.

Proposition 2.2 Suppose that (G1)-(G3) hold. Then:

(1) For any bounded continuous function $f: \mathfrak{X} \rightarrow \mathbb{R}$,

$$
\lim _{t \rightarrow \pm \infty} \omega_{t}(f)=\omega_{ \pm}(f) .
$$

(2) $\sigma \in L^{1}\left(\mathfrak{X}, \mathrm{d} \omega_{ \pm}\right)$and

$$
\omega_{ \pm}(\sigma)=\lim _{t \rightarrow \pm \infty} \omega_{t}(\sigma)=\operatorname{tr}\left(\varsigma\left(D_{ \pm}-D\right)\right)
$$

Note that

$$
\omega_{+}(\sigma)=\lim _{t \rightarrow \infty} \frac{1}{t} \int_{0}^{t} \omega_{s}(\sigma) \mathrm{d} s=-\lim _{t \rightarrow \infty} \frac{1}{t} \operatorname{Ent}\left(\omega_{t} \mid \omega\right) .
$$

We shall call $\omega_{+}$the NESS and the non-negative number $\omega_{+}(\sigma)$ the entropy production of $(\mathfrak{X}, \phi, \omega)$.

\subsection{Entropic fluctuations with respect to the reference state}

Time reversal invariance plays an important role in non-equilibrium statistical mechanics, and in particular in formulation of the fluctuation relations. Hence, we shall also consider the following hypothesis:

(G4) There exists a unitary involution $\vartheta: \mathcal{K} \rightarrow \mathcal{K}$ such that $\vartheta\left(\mathfrak{X}_{l}\right) \subset \mathfrak{X}_{l}, \vartheta \mathcal{L}=-\mathcal{L} \vartheta$, and $\vartheta D=D \vartheta$.

This assumption implies that $D_{-t}=\vartheta D_{t} \vartheta$ for all $t \in \mathbb{R}$, and thus $D_{-}=\vartheta D_{+} \vartheta$ and $\omega_{+}=\omega_{-} \circ \vartheta$. Moreover, it follows from Definition (8) that $\varsigma_{\varsigma}=-\varsigma \vartheta$. This in turn implies that $\operatorname{tr}(D \varsigma)=0$ and

$$
\sigma(x)=(x, \varsigma x), \quad \omega_{+}(\sigma)=-\omega_{-}(\sigma) .
$$

For simplicity of notation and exposition, we shall state and prove our main results under the time reversal invariance assumption, which covers the cases of physical interest. With a minor modifications of the 
statements and the proofs, most of our results hold without this assumption. We leave these generalizations to the interested reader.

The relative Rényi entropy functional, which is defined by

$$
e_{t}(\alpha)=\operatorname{Ent}_{\alpha}\left(\omega_{t} \mid \omega\right)=\log \omega\left(\mathrm{e}^{\alpha \ell_{\omega_{t} \mid \omega}}\right),
$$

is a priori finite only for $\alpha \in[0,1]$. To describe its properties, we introduce the sets

$$
J_{t}=\left\{\alpha \in \mathbb{R} \mid D^{-1}+\alpha T_{t}>0\right\}, \quad t \in \mathbb{R},
$$

and denote by $\mathbb{C}_{ \pm}$the open upper/lower half-plane.

Proposition 2.3 Suppose that $(\mathrm{G} 1)-(\mathrm{G} 4)$ hold. Then:

(1) $J_{t}=\left(-\delta_{t}, 1+\delta_{t}\right)$ for some $\delta_{t} \geq \delta$ and $J_{-t}=J_{t}$.

(2) The function $\alpha \mapsto e_{t}(\alpha)$ is finite on the interval $J_{t}$ and is equal to $+\infty$ for $\alpha \notin J_{t}$. Moreover, this function is convex, extends to an analytic function on the cut plane $\mathbb{C}_{+} \cup \mathbb{C}_{-} \cup J_{t}$, and satisfies

$$
e_{t}(0)=e_{t}(1)=0, \quad e_{t}^{\prime}(0) \leq 0, \quad e_{t}^{\prime}(1) \geq 0 .
$$

In particular, $e_{t}(\alpha) \leq 0$ for $\alpha \in[0,1]$ and $e_{t}(\alpha) \geq 0$ otherwise.

(3) The finite time Evans-Searles symmetry $e_{t}(\alpha)=e_{t}(1-\alpha)$ holds for all $t$ and $\alpha$.

We now study the statistical properties of trajectories as $t \rightarrow+\infty$. The intervals $J_{t}$ do not necessarily form a monotone family, and we define the minimal interval

$$
\underline{J}=\liminf _{t \rightarrow \infty} J_{t}=\bigcup \bigcap_{T>0} J_{t} .
$$

Clearly, one has $\underline{J}=(-\underline{\delta}, 1+\underline{\delta})$, where $\underline{\delta}=\liminf _{t \rightarrow \infty} \delta_{t} \geq \delta$.

Theorem 2.4 Suppose that (G1)-(G4) hold.

(1) The limit

$$
e(\alpha):=\lim _{t \rightarrow+\infty} \frac{1}{t} e_{t}(\alpha)
$$

exists for $\alpha \in \underline{J}$. Moreover, the function $e(\alpha)$ is convex on the interval $\underline{J}$ and satisfies the relations

$$
e(0)=e(1)=0, \quad e^{\prime}(0)=-\omega_{+}(\sigma) \leq 0, \quad e^{\prime}(1)=\omega_{+}(\sigma) \geq 0, \quad e(1-\alpha)=e(\alpha) .
$$

(2) The function $e(\alpha)$ extends to an analytic function on the cut plane $\mathbb{C}_{+} \cup \mathbb{C}_{-} \cup \underline{J}$, and there is a unique signed Borel measure $\nu$ with support contained in $\mathbb{R} \backslash \underline{J}$ such that $\int|r|^{-1} \mathrm{~d}|\nu|(r)<\infty$ and

$$
e(\alpha)=-\int_{\mathbb{R}} \log \left(1-\frac{\alpha}{r}\right) \mathrm{d} \nu(r)
$$

(3) The Large Deviation Principle holds in the following form. The function

$$
I(s)=\sup _{-\alpha \in \underline{J}}(\alpha s-e(-\alpha))
$$

is convex, takes values in $[0, \infty]$, vanishes only at $s=\omega_{+}(\sigma)$, and satisfies the Evans-Searles symmetry relation

$$
I(-s)=I(s)+s \quad \text { for } s \in \mathbb{R} .
$$

Moreover, there is $\varepsilon>0$ such that, for any open set $\mathcal{J} \subset\left(-\omega_{+}(\sigma)-\varepsilon, \omega_{+}(\sigma)+\varepsilon\right)$, we have

$$
\lim _{t \rightarrow \infty} \frac{1}{t} \log \omega\left(\left\{x \in \mathfrak{X} \mid \frac{1}{t} \int_{0}^{t} \sigma_{s}(x) \mathrm{d} s \in \mathcal{J}\right\}\right)=-\inf _{s \in \mathcal{J}} I(s) .
$$


(4) The Central Limit Theorem holds. That is, for any Borel set $B \subset \mathbb{R}$, we have

$$
\lim _{t \rightarrow \infty} \omega\left(\left\{x \in \mathfrak{X} \mid \frac{1}{\sqrt{t}} \int_{0}^{t}\left(\sigma_{s}(x)-\omega_{+}(\sigma)\right) \mathrm{d} s \in B\right\}\right)=\int_{B} \mathrm{e}^{-x^{2} / 2 a} \frac{\mathrm{d} x}{\sqrt{2 \pi a}},
$$

where $a=e^{\prime \prime}(1)$.

(5) The strong law of large numbers holds. That is, for $\omega$-a.e. $x \in \mathfrak{X}$, we have

$$
\lim _{t \rightarrow \infty} \frac{1}{t} \int_{0}^{t} \sigma_{s}(x) \mathrm{d} s=\omega_{+}(\sigma) .
$$

Remark 1. In general, the two limiting measures $\omega_{-}$and $\omega_{+}$are distinct. This property is closely related to the strict positivity of entropy production. In fact, it follows from the second relation in (11) that if $\omega_{-}=\omega_{+}$, then $\omega_{+}(\sigma)=0$ as well as $\omega_{-}(\sigma)=0$, while any of these two conditions imply that the function $e(\alpha)$ vanishes on $[0,1]$ and, hence, identically in view of analyticity.

Remark 2. The representation of $e(\alpha)$ as a logarithmic potential of a signed measure is somewhat surprising, and its mathematical and physical significance remains to be studied in the future. The measure $\nu$ is related to the spectral measure of the operator $Q$ (see the proof of Theorem 2.4 for more details).

Now let $\left\{t_{n}\right\} \subset \mathbb{R}_{+}$be a sequence such that $\delta_{t_{n}} \rightarrow \hat{\delta}$. We define $\hat{J}=(-\hat{\delta}, 1+\hat{\delta})$. Note that, by Proposition 2.3 (1), we have $\hat{\delta} \geq \delta$. In the case when $\hat{\delta}$ coincides with $\bar{\delta}=\lim _{\sup _{t \rightarrow \infty}} \delta_{t}$, we write $\bar{J}$ instead of $\hat{J}$.

Theorem 2.5 Suppose that (G1)-(G4) hold and $\left\{t_{n}\right\} \subset \mathbb{R}_{+}$is a sequence satisfying the above hypothesis.

(1) Let $Q=D_{-}^{1 / 2}\left(D_{-}^{-1}-D_{+}^{-1}\right) D_{-}^{1 / 2}$. Then

$$
-\frac{1}{\bar{\delta}} \leq Q \leq \frac{1}{1+\bar{\delta}}
$$

Furthermore, since the function $g(z)=z^{-1} \log (1-z)$ is analytic in the cut plane $\mathbb{C} \backslash[1, \infty)$, the operator-valued function

$$
E(\alpha)=-\alpha D_{-}^{1 / 2} g(\alpha Q) D_{-}^{1 / 2},
$$

is analytic in the cut plane $\mathbb{C}_{+} \cup \mathbb{C}_{-} \cup \bar{J}$.

(2) For $\alpha \in \hat{J}$, the following relation holds:

$$
\hat{e}(\alpha):=\lim _{n \rightarrow \infty} \frac{1}{t_{n}} e_{t_{n}}(\alpha)=\operatorname{tr}(E(\alpha) \varsigma),
$$

and if $\alpha \in \mathbb{R}$ is not in the closure of $\hat{J}$, then

$$
\limsup _{n \rightarrow \infty} \frac{1}{t_{n}} e_{t_{n}}(\alpha)=\infty
$$

Moreover, the function $\hat{e}(\alpha)$ is convex on the interval $\hat{J}$ and satisfies relations (15).

(3) The Large Deviation Principle holds in the following form. The function

$$
\hat{I}(s)=\sup _{-\alpha \in \hat{J}}(\alpha s-\hat{e}(-\alpha))
$$

is convex, takes values in $[0, \infty]$, vanishes only at $s=\omega_{+}(\sigma)$, and satisfies the Evans-Searles symmetry relation (17). Moreover, for any open interval $\mathcal{J} \subset \mathbb{R}$, we have

$$
\lim _{n \rightarrow \infty} \frac{1}{t_{n}} \log \omega\left(\left\{x \in \mathfrak{X} \mid \frac{1}{t_{n}} \int_{0}^{t_{n}} \sigma_{s}(x) \mathrm{d} s \in \mathcal{J}\right\}\right)=-\inf _{s \in \mathcal{J}} \hat{I}(s) .
$$


Remark 1. The functions $\hat{e}(\alpha)$ constructed in Theorem 2.5 coincide with $e(\alpha)$ on the minimal interval $\underline{J}$. Moreover, by Part (2) of Theorem 2.5, the functions $\hat{e}$ constructed for different sequences $\left\{t_{n}\right\}$ must coincide on the common domain of definition.

Remark 2. If $\bar{\delta}=\infty$, then $\hat{e}(\alpha)=e(\alpha)=0$ for $\alpha \in \mathbb{R}$.

Remark 3. The local Large Deviation Principle described in Part (3) of Theorem 2.4 is an immediate consequence of the local Gärtner-Ellis theorem (see Appendix A.2 in [JOPP]). The global Large Deviation Principle described in Part (3) of Theorem 2.5 cannot be deduced from the Gärtner-Ellis theorem. Our proof of the LDP exploits heavily the Gaussian structure of the model and is motivated by Exercise 2.3.24 in [DZ], see also [BFL, BFR, BD] for related results.

\subsection{Entropic fluctuations with respect to the NESS}

We now turn to the statistical properties of the dynamics under the limiting measures $\omega_{ \pm}$. In view of the time-reversal invariance (G4), it suffices to study the case of one of these measures, and we shall restrict ourselves to $\omega_{+}$. Let us set (cf. Part (2) of Proposition 2.1)

$$
e_{t+}(\alpha)=\log \omega_{+}\left(\mathrm{e}^{-\alpha \ell_{\omega_{t} \mid \omega}}\right)=\log \omega_{+}\left(\mathrm{e}^{-\alpha \int_{0}^{t} \sigma_{-s} \mathrm{~d} s}\right)=\log \omega_{+}\left(\mathrm{e}^{-\alpha \int_{0}^{t} \sigma_{s} \mathrm{~d} s}\right),
$$

where the last relation follows from the invariance of $\omega_{+}$under the flow $\phi^{t}$. Note that, a priori, $e_{t+}(\alpha)$ might not be finite for any $\alpha \neq 0$.

Theorem 2.6 Suppose that (G1)-(G4) hold. Then:

(1) For any $t \in \mathbb{R}$, the function $\mathbb{R} \ni \alpha \mapsto e_{t+}(\alpha) \in(-\infty,+\infty]$ is convex.

(2) The set

$$
J_{t}^{+}=\left\{\alpha \in \mathbb{R} \mid D_{+}^{-1}-\alpha T_{t}>0\right\}
$$

is an open interval containing $(-\delta, \delta)$, and the function $e_{t+}(\alpha)$ is real analytic on $J_{t}^{+}$and takes value $+\infty$ on its complement.

(3) Let $\underline{J}^{+}$be the interior of the set

$$
\liminf _{t \rightarrow \infty} J_{t}^{+}=\bigcup_{T>0} \bigcap_{t>T} J_{t}^{+} .
$$

Then $\underline{J}^{+}$is an open interval containing $(-\delta, \delta)$. Moreover, for $\alpha \in \underline{J}^{+}$, the limit

$$
e_{+}(\alpha)=\lim _{t \rightarrow \infty} \frac{1}{t} e_{t+}(\alpha)
$$

exists and defines a real-analytic function on $\underline{J}^{+}$. Finally, if $\alpha$ is not in the closure of $\underline{J}^{+}$, then

$$
\limsup _{t \rightarrow \infty} \frac{1}{t} e_{t+}(\alpha)=+\infty \text {. }
$$

(4) The Large Deviation Principle holds in the following form. The function

$$
I^{+}(s)=\sup _{-\alpha \in \underline{J}^{+}}\left(\alpha s-e_{+}(-\alpha)\right)
$$

is convex, takes values in $[0, \infty]$, and vanishes only at $s=\omega_{+}(\sigma)$. Moreover, there is an open interval $\mathbb{I}^{+}$containing $\omega_{+}(\sigma)$ such that, for any open set $\mathcal{J} \subset \mathbb{I}^{+}$,

$$
\lim _{t \rightarrow \infty} \frac{1}{t} \log \omega_{+}\left(\left\{x \in \mathfrak{X} \mid \frac{1}{t} \int_{0}^{t} \sigma_{s}(x) \mathrm{d} s \in \mathcal{J}\right\}\right)=-\inf _{s \in \mathcal{J}} I^{+}(s) .
$$


(5) The Central Limit Theorem holds. That is, for any Borel set $B \subset \mathbb{R}$,

$$
\lim _{t \rightarrow \infty} \omega_{+}\left(\left\{x \in \mathfrak{X} \mid \frac{1}{\sqrt{t}} \int_{0}^{t}\left(\sigma_{s}(x)-\omega_{+}(\sigma)\right) \mathrm{d} s \in B\right\}\right)=\int_{B} \mathrm{e}^{-x^{2} / 2 a_{+}} \frac{\mathrm{d} x}{\sqrt{2 \pi a_{+}}},
$$

where $a_{+}=e_{+}^{\prime \prime}(0)$.

(6) The strong law of large numbers holds. That is, for $\omega_{+}$-a.e. $x \in \mathfrak{X}$, we have

$$
\lim _{n \rightarrow \infty} \frac{1}{t} \int_{0}^{t} \sigma_{s}(x) \mathrm{d} s=\omega_{+}(\sigma) .
$$

(7) Let $\underline{J}$ be as in Theorem 2.4. Then $e_{+}(\alpha)=e(\alpha)$ for $\alpha \in \underline{J}^{+} \cap \underline{J}$. Moreover, there is an open interval $\mathbb{J}^{+} \subset \mathbb{I}^{+}$such that $I^{+}(s)=I(s)$ for $s \in \mathbb{J}^{+}$.

Remark. This theorem is a refinement of Proposition 9.5 in [JPR]. We point out that parts (1) and (3) of that proposition are inaccurately formulated: in part (1), the interval $(-\delta, 1+\delta)$ has to be replaced with $(-\delta, \delta)$, while in part (3) the interval $\left(-\langle\sigma\rangle_{+}-\varepsilon,\langle\sigma\rangle_{+}+\varepsilon\right)$ has to be replaced with $\left(\langle\sigma\rangle_{+}-\varepsilon,\langle\sigma\rangle_{+}+\varepsilon\right)$.

Finally, we have the following analogue of Theorem 2.5 on statistical properties of the dynamics under the limiting measure $\omega_{+}$. Let $\left\{t_{n}\right\} \subset \mathbb{R}_{+}$be an arbitrary increasing sequence going to $+\infty$ such that the intervals $J_{t_{n}}^{+}$defined by (26) converge to a limiting interval $\hat{J}^{+}$.

Theorem 2.7 Under the hypotheses of Theorem 2.6 the following assertions hold.

(1) For $\alpha \in \hat{J}^{+}$, the limit

$$
\hat{e}_{+}(\alpha):=\lim _{n \rightarrow \infty} \frac{1}{t_{n}} e_{t_{n}+}(\alpha)
$$

exists and defines a real-analytic function on $\hat{J}^{+}$. If $\alpha$ does not belong to the closure of $\hat{J}^{+}$, then

$$
\limsup _{n \rightarrow \infty} \frac{1}{t_{n}} e_{t_{n}+}(\alpha)=\infty
$$

Moreover, $\hat{e}_{+}(\alpha)$ and $\operatorname{tr}(E(\alpha) \varsigma)$ coincide on their common domain of definition.

(2) The Large Deviation Principle holds in the following form. The function

$$
\hat{I}^{+}(s)=\sup _{-\alpha \in \hat{J}^{+}}\left(\alpha s-\hat{e}_{+}(-\alpha)\right)
$$

is convex, takes values in $[0, \infty]$ and vanishes only at $s=\omega_{+}(\sigma)$. Moreover, for any open interval $\mathcal{J} \subset \mathbb{R}$, we have

$$
\lim _{n \rightarrow \infty} \frac{1}{t_{n}} \log \omega_{+}\left(\left\{x \in \mathfrak{X} \mid \frac{1}{t_{n}} \int_{0}^{t_{n}} \sigma_{s}(x) \mathrm{d} s \in \mathcal{J}\right\}\right)=-\inf _{s \in \mathcal{J}} \hat{I}^{+}(s) .
$$

The proof of this result is completely similar to that of Theorem 2.5, and therefore we omit it.

Remark. Unlike in the case of the Evans-Searles symmetry, there is no a priori reason why the limiting intervals $\hat{J}^{+}$should be symmetric around $\alpha=\frac{1}{2}$, and indeed in all cases we know where $\hat{J}^{+}$can be computed, this property does not hold. Hence, the relation $\hat{e}_{+}(\alpha)=\hat{e}_{+}(1-\alpha)$ may fail since one side may be finite and the other infinite, leading to the failure of the Gallavotti-Cohen symmetry $\hat{I}^{+}(-s)=\hat{I}^{+}(s)+s$. The fact that for unbounded entropy production observables the Gallavotti-Cohen symmetry may fail is known in the physics literature [BaCo, BGGZ, BJMS, Fa, HRS, Vi1, Vi2, ZC]. In these works one can also find various prescriptions how the entropy production observable can be modified so that the GallavottiCohen symmetry is restored. We shall discuss this topic in the continuation of this paper [JPS]. 


\subsection{Perturbations}

We shall consider the following type of perturbation of the reference state $\omega$. Let $P$ be a bounded selfadjoint operator on $\mathcal{K}$ such that $D^{-1}+P>0$. To avoid trivialities, we assume that $P$ is not the zero operator. Let

$$
D^{P}=\left(D^{-1}+P\right)^{-1}
$$

and let $\omega^{P}$ be the centered Gaussian measure with covariance $D^{P}$. Obviously,

$$
D_{t}^{P}=\left(D_{t}^{-1}+P_{t}\right)^{-1},
$$

where $P_{t}=\mathrm{e}^{-t \mathcal{L}^{*}} P \mathrm{e}^{-t \mathcal{L}}$. We consider the following two cases, assuming that (G1)-(G4) hold for $D$.

Case 1. $P$ is a non-negative trace class operator such that $\vartheta P=P \vartheta$, and $\underset{t \rightarrow \pm \infty}{\operatorname{sim}} P_{t}=0$.

In this case, $\omega^{P}$ and $\omega$ are equivalent and (G1)-(G4) also hold for $D^{P}$. Moreover, using the superscript $P$ to denote the objects associated with the initial measure $\omega^{P}$, we easily check that

$$
D_{ \pm}^{P}=D_{ \pm}, \quad E^{P}(\alpha)=E(\alpha), \quad \varsigma^{P}=\varsigma+\frac{1}{2}\left(\mathcal{L}^{*} P+P \mathcal{L}\right), \quad \omega_{+}^{P}\left(\sigma^{P}\right)=\omega_{+}(\sigma),
$$

where we used (21) to derive the second relation. We also see that the functions $e^{P}(\alpha)$ and $e(\alpha)$ coincide on $\underline{J} \cap \underline{J}^{P}$. It is possible, however, that $\underline{J}^{P} \neq \underline{J}$ and $\underline{J}^{+P} \neq \underline{J}^{+}$, and in fact the difference could be quite dramatic. Indeed, let us fix $P$ and consider the perturbation $\lambda P$ for $\lambda>0$. Pick a unit vector $\varphi$ such that $P \varphi=e \varphi$ with $e>0$.

We consider first the case of $\underline{J}^{\lambda P}$. One easily sees that for any $\alpha>1$,

$$
\left(\varphi,\left(\left(D^{\lambda P}\right)^{-1}+\alpha T_{t}^{\lambda P}\right) \varphi\right) \leq \frac{\alpha}{m}-\lambda\left((\alpha-1) e-\alpha\left(\varphi, P_{t} \varphi\right)\right) .
$$

There exists $t_{0}$ such that for $t>t_{0},(\alpha-1) e-\alpha\left(\varphi, P_{t} \varphi\right)>(\alpha-1) e / 2$. Hence, for $t>t_{0}$ and $\lambda>2 \alpha / e m(\alpha-1)$ the right hand side of (30) is negative which implies that $\alpha>1+\delta_{t}^{\lambda P}$. Thus

$$
\underline{\delta}^{\lambda P}=\liminf _{t \rightarrow \infty} \delta_{t}^{\lambda P} \leq \alpha-1
$$

provided $\lambda>2 \alpha / e m(\alpha-1)$. Letting now $\alpha \downarrow 1$ we conclude that

$$
\lim _{\lambda \rightarrow \infty} \underline{\delta}^{\lambda P}=0
$$

and the intervals $\underline{J}^{\lambda P}$ collapse to $[0,1]$ in the limit $\lambda \rightarrow \infty$.

To deal with the case of $\underline{J}^{+\lambda P}$, we set $\psi_{\alpha, t}=\mathrm{e}^{t \mathcal{L}} \varphi$ for $\alpha>0$ and $\psi_{\alpha, t}=\varphi$ for $\alpha<0$. A simple analysis yields

$$
\left(\psi_{\alpha, t},\left(\left(D_{+}^{\lambda P}\right)^{-1}-\alpha T_{t}^{\lambda P}\right) \psi_{\alpha, t}\right) \leq \frac{1+|\alpha|}{m}\left\|\psi_{\alpha, t}\right\|^{2}-\lambda|\alpha|\left(e-\left(\varphi, P_{t} \varphi\right)\right) .
$$

Repeating the previous argument, one shows that the length of the interval $\underline{J}^{+\lambda P}$ goes to zero as $\lambda \rightarrow \infty$, so that the intervals $\underline{J}^{+\lambda P}$ collapse to $\{0\}$.

Case 2. $P>0, \vartheta P=P \vartheta$, and $P_{t}=P$ for all $t \in \mathbb{R}$.

Hypotheses $(\mathrm{G} 1)-(\mathrm{G} 4)$ again hold for $D^{P}$, and we have

$$
D_{+}^{P}=\left(D_{+}^{-1}+P\right)^{-1}, \quad \varsigma^{P}=\varsigma, \quad \sigma^{P}=\sigma .
$$

Replacing $P$ with $\lambda P$, it is easy to see that $\delta^{\lambda P}$, defined by (10), satisfies $\lim _{\lambda \rightarrow \infty} \delta^{\lambda P}=\infty$. Since $\left(-\delta^{\lambda P}, 1+\delta^{\lambda P}\right) \subset \underline{J}^{\lambda P}$ and $\left(-\delta^{\lambda P}, \delta^{\lambda P}\right) \subset \underline{J}^{+\lambda P}$, we see that the intervals $\underline{J}^{\lambda P}$ and $\underline{J}^{+\lambda P}$ extend to the whole real line in the limit $\lambda \rightarrow \infty$. 


\section{Examples}

\subsection{Toy model}

Suppose that the generator $\mathcal{L}$ satisfies $\mathcal{L}^{*}=-\mathcal{L}$, and let $\varphi \in \mathcal{K}$ be a unit vector such that the spectral measure for $\mathcal{L}$ and $\varphi$ is purely absolutely continuous. Let

$$
D=I+\lambda P_{\varphi}
$$

where $P_{\varphi}=(\varphi, \cdot) \varphi$ and $\lambda>-1$. Then $D_{t}=I+\lambda P_{\varphi_{t}}$, where $\varphi_{t}=\mathrm{e}^{t \mathcal{L}} \varphi$ is a continuous curve of unit vectors converging weakly to zero as $t \rightarrow+\infty$. Let $\lambda_{ \pm}=\frac{1}{2}(|\lambda| \pm \lambda)$ denote the positive/negative part of $\lambda$. One easily verifies that (G1)-(G3) hold with $m=1-\lambda_{-}, M=1+\lambda_{+}$and $D_{ \pm}=I$, so that

$$
\delta=\left|\frac{1}{2}+\frac{1}{\lambda}\right|-\frac{1}{2}
$$

Without loss of generality we may assume that (G4) holds. ${ }^{2}$ Since $\left(I+\lambda P_{\psi}\right)^{-1}=I-\frac{\lambda}{1+\lambda} P_{\psi}$ for any unit vector $\psi$ and any $\lambda \neq-1$, we have

$$
\begin{aligned}
& D^{-1}+\alpha T_{t}=I-\frac{\lambda}{1+\lambda}\left((1-\alpha) P_{\varphi}+\alpha P_{\varphi_{t}}\right), \\
& D_{+}^{-1}-\alpha T_{t}=I-\frac{\lambda}{1+\lambda} \alpha\left(P_{\varphi}-P_{\varphi_{t}}\right) .
\end{aligned}
$$

Using the simple fact that for any two linearly independent unit vectors $\varphi, \psi$ and all $a, b \in \mathbb{R}$,

$$
\operatorname{sp}\left(a P_{\varphi}+b P_{\psi}\right)=\{0\} \cup\left\{\frac{a+b}{2} \pm \sqrt{\left(\frac{a-b}{2}\right)^{2}+a b(\psi, \varphi)^{2}}\right\},
$$

one easily shows that

$$
\delta_{t}=\sqrt{\frac{1}{4}+\frac{1+\lambda}{\lambda^{2}\left(1-\left(\varphi, \varphi_{t}\right)^{2}\right)}}-\frac{1}{2}, \quad J_{t}^{+}=\left\{\alpha \in \mathbb{R}|| \alpha \mid<\frac{1+\lambda}{|\lambda| \sqrt{1-\left(\varphi, \varphi_{t}\right)^{2}}}\right\} .
$$

Recalling that $\left(\varphi, \varphi_{t}\right) \rightarrow 0$ as $t \rightarrow+\infty$ we see that for all $\lambda>-1, \underline{\delta}=\bar{\delta}=\delta$ and $\underline{J}^{+}=\left(-\delta^{+}, \delta^{+}\right)$where

$$
\delta^{+}=\frac{1+\lambda}{|\lambda|}=\left\{\begin{array}{cl}
\delta & \text { for } \quad \lambda \in(-1,0], \\
1+\delta & \text { for } \quad \lambda \in[0, \infty)
\end{array}\right.
$$

Furthermore, evaluating Relations (46) and (86) established below, we obtain

$$
\begin{aligned}
e_{t}(\alpha) & =-\frac{1}{2} \log \left(1+\frac{\lambda^{2}}{1+\lambda} \alpha(1-\alpha)\left(1-\left(\varphi, \varphi_{t}\right)^{2}\right)\right), \\
e_{t+}(\alpha) & =-\frac{1}{2} \log \left(1-\frac{\lambda^{2}}{(1+\lambda)^{2}} \alpha^{2}\left(1-\left(\varphi, \varphi_{t}\right)^{2}\right)\right) .
\end{aligned}
$$

It follows that

$$
\lim _{t \rightarrow \infty} \frac{1}{t} e_{t}(\alpha)=\left\{\begin{array}{lll}
0 & \text { for } & \left|\alpha-\frac{1}{2}\right|<\frac{1}{2}+\delta, \\
+\infty & \text { for } \quad\left|\alpha-\frac{1}{2}\right|>\frac{1}{2}+\delta,
\end{array} \quad \lim _{t \rightarrow \infty} \frac{1}{t} e_{t+}(\alpha)=\left\{\begin{array}{lll}
0 & \text { for } & |\alpha|<\delta^{+} \\
+\infty & \text { for } & |\alpha|>\delta^{+}
\end{array}\right.\right.
$$

Finally, one easily compute the Legendre transforms of these limiting functions,

$$
I(s)=\left(\frac{1}{2}+\delta\right)|s|-\frac{1}{2} s, \quad I^{+}(s)=\delta^{+}|s| .
$$

While the first one satisfies the fluctuation relation, i.e., $I(s)+\frac{1}{2} s$ is an even function, the second one does not.

${ }^{2}$ That can be always achieved by replacing $\mathcal{K}$ with $\mathcal{K} \oplus \mathcal{K}, \mathcal{L}$ with $\mathcal{L} \oplus \mathcal{L}^{*}, \varphi$ with $\frac{1}{\sqrt{2}} \varphi \oplus \varphi$, and setting $\vartheta\left(\psi_{1} \oplus \psi_{2}\right)=\psi_{2} \oplus \psi_{1}$. 


\subsection{One-dimensional crystal}

We follow [JOPP] and consider the simplest example of the one-dimensional harmonic crystal. If $\Lambda \subset \mathbb{Z}$ is the crystal lattice, then the phase space and Hamiltonian of the harmonic crystal are

$$
\begin{gathered}
\mathbb{R}^{\Lambda} \oplus \mathbb{R}^{\Lambda}=\left\{(p, q)=\left(\left\{p_{n}\right\}_{n \in \Lambda},\left\{q_{n}\right\}_{n \in \Lambda}\right) \mid p_{n}, q_{n} \in \mathbb{R}\right\}, \\
H_{\Lambda}(p, q)=\sum_{n \in \Lambda}\left(\frac{p_{n}^{2}}{2}+\frac{q_{n}^{2}}{2}+\frac{\left(q_{n}-q_{n-1}\right)^{2}}{2}\right),
\end{gathered}
$$

where we set $q_{k}=0$ for $k \notin \Lambda$ (Dirichlet boundary conditions). The Hamilton equation of motions are

$$
\left(\begin{array}{c}
\dot{p} \\
\dot{q}
\end{array}\right)=\mathcal{L}_{\Lambda}\left(\begin{array}{c}
p \\
q
\end{array}\right)
$$

where

$$
\mathcal{L}_{\Lambda}=\left(\begin{array}{cc}
0 & -j_{\Lambda} \\
1_{\Lambda} & 0
\end{array}\right)
$$

$j_{\Lambda}$ is the restriction of the finite difference operator

$$
(j q)_{n}=3 q_{n}-q_{n+1}-q_{n-1}
$$

to $\mathbb{R}^{\Lambda}$ with Dirichlet boundary condition and $1_{\Lambda}$ the identity on $\mathbb{R}^{\Lambda}$ (which we shall later identify with the projection $\mathbb{R}^{\mathbb{Z}} \rightarrow \mathbb{R}^{\Lambda}$ ). Clearly, for all $\Lambda, j_{\Lambda}$ is a bounded selfadjoint operator on $\ell_{\mathbb{R}}^{2}(\Lambda)$ satisfying $1 \leq j_{\Lambda} \leq 5$.

To fit this model into our abstract framework, we set $\Gamma_{\Lambda}=\Lambda \times \mathbb{Z}_{2}, \mathfrak{X}_{\Lambda}=\mathbb{R}^{\Gamma_{\Lambda}}=\mathbb{R}^{\Lambda} \oplus \mathbb{R}^{\Lambda}$ with the weight sequence $l=\left(l_{n, i}\right)_{(n, i) \in \Gamma_{\Lambda}}$, where $l_{n, i}=c_{\Lambda}\left(1+n^{2}\right)^{-1}$ and $c_{\Lambda}$ is a normalization constant. One easily verifies that $\mathcal{L}_{\Lambda}^{*} \mathfrak{X}_{\Lambda l}^{*} \subset \mathfrak{X}_{\Lambda l}^{*}$ and the dynamics of the harmonic crystal is described by the group $\mathrm{e}^{t \mathcal{L}_{\Lambda}}$. Let $h_{\Lambda}$ be the self-adjoint operator on $\mathcal{K}_{\Lambda}=\ell_{\mathbb{R}}^{2}(\Lambda) \oplus \ell_{\mathbb{R}}^{2}(\Lambda)$ associated to the quadratic form $2 H_{\Lambda}$. Energy conservation implies $\mathcal{L}_{\Lambda}^{*} h_{\Lambda}+h_{\Lambda} \mathcal{L}_{\Lambda}=0$. Equivalently, the operator $L_{\Lambda}$ defined by

$$
L_{\Lambda}=h_{\Lambda}^{1 / 2} \mathcal{L}_{\Lambda} h_{\Lambda}^{-1 / 2}=\left(\begin{array}{cc}
0 & -j_{\Lambda}^{1 / 2} \\
j_{\Lambda}^{1 / 2} & 0
\end{array}\right),
$$

is skew-adjoint. Since $1 \leq h_{\Lambda} \leq 5$, this implies in particular that the group $\mathrm{e}^{t \mathcal{L}_{\Lambda}}$ is uniformly bounded on $\mathcal{K}_{\Lambda}$.

Our starting point is harmonic crystal on $\Lambda=\mathbb{Z}$ and in this case we drop the subscript $\Lambda$. For our purposes we will view this crystal as consisting of three parts, the left, central, and right, specified by

$$
\Lambda_{\ell}=(-\infty,-1], \quad \Lambda_{c}=\{0\}, \quad \Lambda_{r}=[1, \infty) .
$$

In what follows we, adopt the shorthands $H_{\Lambda_{\ell}}=H_{\ell}, h_{\Lambda_{\ell}}=h_{\ell}, j_{\Lambda_{\ell}}=j_{\ell}$, etc. Clearly

$$
\mathfrak{X}=\mathfrak{X}_{\ell} \oplus \mathfrak{X}_{c} \oplus \mathfrak{X}_{r}, \quad \mathcal{K}=\mathcal{K}_{\ell} \oplus \mathcal{K}_{c} \oplus \mathcal{K}_{r},
$$

where $\mathcal{K}_{s}=\ell_{\mathbb{R}}^{2}\left(\Lambda_{s}\right) \oplus \ell_{\mathbb{R}}^{2}\left(\Lambda_{s}\right)$ for $s=\ell, c, r$, and

$$
H=H_{0}+V_{\ell}+V_{r},
$$

where

$$
H_{0}=H_{\ell}+H_{c}+H_{r}
$$

and $V_{\ell}(p, q)=-q_{0} q_{-1}, V_{r}(p, q)=-q_{0} q_{1}$.

The reference state $\omega$ is the centered Gaussian measure with covariance

$$
D=D_{\ell} \oplus D_{c} \oplus D_{r}
$$


where

$$
D_{s}=T_{s}\left(\begin{array}{cc}
I_{s} & 0 \\
0 & j_{s}^{-1}
\end{array}\right), \quad s=\ell, c, r,
$$

$I_{s}$ is the identity on $\ell_{\mathbb{R}}^{2}\left(\Lambda_{s}\right)$, and $T_{s}>0$. Thus, initially the left/right part of the crystal are in thermal equilibrium at temperature $T_{\ell / r}$. The Hamiltonian $V_{\ell / r}$ couples the left/right part of the crystal to the oscillator located at the site $n=0$ and this allows for the transfer of the energy/entropy between these two parts. The entropic fluctuation theorems for this particular Gaussian dynamical system concern statistics of the energy/entropy flow between the left and right parts of the crystal.

Hypothesis (G1)-(G4) are easily verified following the arguments of Chapter 1 in the lecture notes [JOPP] and one finds that

$$
\omega_{+}(\sigma)=\kappa \frac{\left(T_{\ell}-T_{r}\right)^{2}}{T_{\ell} T_{r}},
$$

where $\kappa=(\sqrt{5}-1) / 2 \pi$, and

$$
e(\alpha)=-\kappa \log \left(1+\frac{\left(T_{\ell}-T_{r}\right)^{2}}{T_{\ell} T_{r}} \alpha(1-\alpha)\right) .
$$

Note that $e(\alpha)$ is finite on the interval $J_{o}=\left(-\delta_{o}, 1+\delta_{o}\right)$, where

$$
\delta_{o}=\frac{\min \left(T_{\ell}, T_{r}\right)}{\left|T_{\ell}-T_{r}\right|}
$$

and takes the value $+\infty$ outside the interval $J_{o}$. Note also that $\delta_{o}$ can take any value in $(0, \infty)$ for appropriate choices of $T_{\ell}, T_{r} \in(0, \infty)$. The measure $\nu$ in Part (2) of Theorem 2.4 is

$$
\nu=\kappa \mathfrak{D}_{-\delta_{o}}+\kappa \mathfrak{D}_{1+\delta_{o}},
$$

where $\mathfrak{D}_{a}$ is the Dirac measure centered at $a$.

We finish this section with several remarks.

Remark 1. The intervals $\underline{J}, \underline{J}^{+}$can be strictly smaller then $J_{o}$. To see this, fix $T_{c}, \delta_{o}, \alpha>1$, and set $T_{r}=\left(1+\delta_{o}^{-1}\right) T_{\ell}$ to ensure Relation (33). Let $\varphi \in \mathcal{K}$ be such that $\left(\varphi, h_{c} \varphi\right)=1$. One has

$$
\left(\varphi,\left(D^{-1}+\alpha T_{t}\right) \varphi\right)=\sum_{s} \frac{1}{T_{s}}\left((1-\alpha)\left(\varphi, h_{s} \varphi\right)+\alpha\left(\varphi_{t}, h_{s} \varphi_{t}\right)\right)
$$

where $\varphi_{t}=\mathrm{e}^{-t \mathcal{L}} \varphi$. Since the skew-adjoint operator $L$ has purely absolutely continuous spectrum and $h_{c}$ is compact, there exists $t_{0}>0$ such that

$$
\left(\varphi_{t}, h_{c} \varphi_{t}\right)=\left(\mathrm{e}^{-t L} h^{1 / 2} \varphi, h^{-1 / 2} h_{c} h^{-1 / 2} \mathrm{e}^{-t L} h^{1 / 2} \varphi\right)<\frac{\alpha-1}{2 \alpha}
$$

for all $t>t_{0}$. Moreover, since the Hamiltonian flow is uniformly bounded there exists a constant $C$ such that

$$
\frac{1}{T_{\ell / r}}\left((1-\alpha)\left(\varphi, h_{\ell / r} \varphi\right)+\alpha\left(\varphi_{t}, h_{\ell / r} \varphi_{t}\right)\right) \leq C \frac{\alpha}{T_{\ell}} .
$$

Summing up, if $T_{\ell}>4 C T_{c} \alpha /(\alpha-1)$, then

$$
\left(\varphi,\left(D^{-1}+\alpha T_{t}\right) \varphi\right) \leq \frac{1-\alpha}{2 T_{c}}+2 C \frac{\alpha}{T_{\ell}}<0,
$$

for all $t>t_{0}$ and hence $\underline{\delta}<\alpha$. Thus, in the limit $T_{\ell} \rightarrow \infty$ the interval $\underline{J}$ collapses to $[0,1]$. In a similar way one can show that in the same limit the interval $\underline{J}^{+}$collapses to $\{0\}$. On the other hand, arguing as in the Case 2 of Section 2.6, one can always take $T_{\ell / r}, T_{c} \rightarrow 0$ in such a way that in this limit the intervals $\underline{J}$, $\underline{J}^{+}$extend to the whole real line. 
Remark 2. Somewhat surprisingly, even in the simplest example of the harmonic crystal discussed in this section, it appears difficult to effectively estimate the location of the intervals $\underline{J}, \underline{J}^{+}$outside of the perturbative regimes. In particular, the subtleties regarding the location of these sets were overlooked in Sections $1.11,1.14$ and 1.15 of the lecture notes [JOPP]. These difficulties raise many interesting questions and we leave the complete analysis of these aspects as an open problem.

Remark 3. An interesting question is whether one can find $P$ such that for the perturbed reference state $\omega^{P}$ as defined in Section 2.6 one has $\underline{J}=J_{o}$. That can be done as follows. Set $\beta_{s}=1 / T_{s}$, suppose that $\beta_{r}>\beta_{\ell}$ and let

$$
P=\left(\begin{array}{cc}
\left(\beta_{r}-\beta_{c}\right) 1_{c} & 0 \\
0 & \left(\beta_{r}+2 \beta_{\ell}-3 \beta_{c}\right) j_{c}+\beta_{\ell} v_{\ell}+\beta_{r} v_{r}
\end{array}\right),
$$

where $v_{\ell / r}$ denotes the selfadjoint operator associated with the quadratic form $2 V_{\ell / r}$. One easily checks that

$$
D^{P}=\left(\beta_{r} h-X h_{\ell}^{(N)}\right)^{-1},
$$

where $X=\beta_{r}-\beta_{\ell}>0$,

$$
h_{\ell}^{(N)}=\left(\begin{array}{cc}
1_{\Lambda_{\ell} \cup \Lambda_{c}} & 0 \\
0 & j_{\ell}^{(N)}
\end{array}\right)
$$

and $j_{\ell}^{(N)}$ denotes the restriction of the operator (31) to $\mathbb{R}^{\Lambda_{\ell} \cup \Lambda_{c}}$ with Neumann boundary condition. We are concerned with the interval

$$
J_{t}^{P}=\left\{\alpha \in \mathbb{R} \mid\left(D^{P}\right)^{-1}+\alpha T_{t}^{P}>0\right\} .
$$

Since

$$
\left(D_{t}^{P}\right)^{-1}=\beta_{r} h-X \mathrm{e}^{-t \mathcal{L}^{*}} h_{\ell}^{(N)} \mathrm{e}^{-t \mathcal{L}}=h^{1 / 2}\left(\beta_{r}-X \mathrm{e}^{t L} h^{-1 / 2} h_{\ell}^{(N)} h^{-1 / 2} \mathrm{e}^{-t L}\right) h^{1 / 2}
$$

a simple computation gives

$$
\left(D^{P}\right)^{-1}+\alpha T_{t}^{P}=h^{1 / 2}\left(\beta_{r}-(1-\alpha) X h^{-1 / 2} h_{\ell}^{(N)} h^{-1 / 2}-\alpha X \mathrm{e}^{t L} h^{-1 / 2} h_{\ell}^{(N)} h^{-1 / 2} \mathrm{e}^{-t L}\right) h^{1 / 2},
$$

and hence

$$
J_{t}^{P}=\left\{\alpha \in \mathbb{R} \mid \beta_{r} / X>(1-\alpha) h^{-1 / 2} h_{\ell}^{(N)} h^{-1 / 2}+\alpha \mathrm{e}^{t L} h^{-1 / 2} h_{\ell}^{(N)} h^{-1 / 2} \mathrm{e}^{-t L}\right\} .
$$

Since $\beta_{r} / X=1+\delta_{o}$ and

$$
0 \leq h_{\ell}^{(N)} \leq h
$$

we have that for all $t$,

$$
\left(-\delta_{o}, 1+\delta_{o}\right) \subset J_{t}^{P}
$$

Thus, $\lim _{t \rightarrow \infty} \delta_{t}^{P}=\delta_{o}$ and $\underline{J}^{P}=J_{o}$.

Remark 4. In contrast to Remark 3, we do not know whether there exists $P$ such that for the perturbed reference state $\omega^{P}$ one has $\underline{J}^{+P}=J_{o}$.

Remark 5. In the equilibrium case $T_{\ell}=T_{r}=T$ we have $\omega_{+}(\sigma)=0$, and one may naively expect that $\sigma$ does not fluctuate with respect to $\omega$ and $\omega_{+}$, i.e., that $e(\alpha)=e_{+}(\alpha)=0$ for all $\alpha$, and that $I(s)=I^{+}(s)=\infty$ if $s \neq 0$. If one also takes $T_{c}=T$ and the perturbed reference state described in Remark 3, then $\sigma=0$, and the above expectation is obviously correct. On the other hand, for the reference state determined by $D$, in the high-temperature regime $T \rightarrow \infty, T_{c}$ fixed, the interval $J$ collapses to $[0,1]$ while the interval $\underline{J}^{+}$collapses to $\{0\}$. Hence, in this regime, the rate functions $\hat{I}(s)$ and $\hat{I}^{+}(s)$ are linear for $s \leq 0$ and $s \geq 0$, with the slopes of the linear parts determined by the end points of the finite intervals $\hat{J}$ and $\hat{J}^{+}$, and the entropy production observable has non-trivial fluctuations.

Remark 6. The scattering theory arguments of [JOPP] that lead to the derivation of the formula (32) extend to the case of inhomogeneous one-dimensional harmonic crystal with Hamiltonian

$$
H_{\Lambda}(p, q)=\sum_{n \in \Lambda}\left(\frac{p_{n}^{2}}{2}+\frac{\omega_{n} q_{n}^{2}}{2}+\frac{\kappa_{n}\left(q_{n}-q_{n-1}\right)^{2}}{2}\right),
$$


where $\omega_{n}$ and $\kappa_{n}$ are positive numbers satisfying

$$
C^{-1} \leq \omega_{n}, \kappa_{n} \leq C \text { for all } n \in \mathbb{Z}
$$

and $C \geq 1$ is a constant. In this case the operator $j$ is the Jacobi matrix

$$
(j q)_{n}=\left(\omega_{n}+\kappa_{n}+\kappa_{n+1}\right) q_{n}-\kappa_{n} q_{n-1}-\kappa_{n+1} q_{n+1}, \quad n \in \mathbb{Z} .
$$

One easily verifies that Hypotheses (G1), (G2), and (G4) hold. If $j$ has absolutely continuous spectrum (considered as a self-adjoint operator on $\ell_{\mathbb{C}}^{2}(\mathbb{Z})$ ), then $(\mathrm{G} 3)$ also holds. Moreover, $\omega_{+}(\sigma)$ and $e(\alpha)$ can be computed in closed form in terms of the scattering data of the pair $\left(j, j_{0}\right)$, where $j_{0}=j_{\ell} \oplus j_{c} \oplus j_{r}$ (for related computations in the context of open quasi-free quantum systems we refer the reader to [JLP, JOPP, Lan]). The formulas for $\omega_{+}(\sigma)$ and $e(\alpha)$ involve the scattering matrix of the pair $\left(j, j_{0}\right)^{3}$ and estimating the location of the intervals $\underline{J}, \underline{J}^{+}$is difficult. However, the interesting aspect of the formula for $e(\alpha)$ is that it allows to express the measure $\nu$ in Part (2) of Theorem 2.4 in terms of the scattering data. The mathematical and physical significance of this representation remain to be studied in the future. Finally, the scattering methods can be extended to treat an arbitrary number of infinite harmonic reservoirs coupled to a finite harmonic system. The discussion of such extensions is beyond the scope of this paper.

\section{Proofs}

\subsection{An auxiliary lemma}

Using the notation and conventions of Section 2.1, we have the following simple result.

Lemma 4.1 (1) If $A=A^{*} \in \mathcal{T}$, then the quadratic form $\ell_{\mathbb{R}}^{2}(\Gamma) \ni x \mapsto q_{A}(x)=(x$, Ax) has a unique extension to an element of $L^{1}\left(\mathfrak{X}, \mathrm{d} \omega_{D}\right)$ with a norm satisfying $\left\|q_{A}\right\|_{1} \leq\|D\|\|A\|_{1}$. Moreover,

$$
\int q_{A}(x) \mathrm{d} \omega_{D}(x)=\operatorname{tr}(D A)
$$

(2) Let $\mathbb{R} \ni t \mapsto A_{t}=A_{t}^{*} \in \mathcal{T}$ be differentiable at $t=t_{0}$ and let $\dot{A}_{t_{0}}$ be its derivative. Then the map $\mathbb{R} \ni t \mapsto q_{A_{t}} \in L^{1}\left(\mathfrak{X}, \mathrm{d} \omega_{D}\right)$ is differentiable at $t=t_{0}$ and

$$
\left.\frac{\mathrm{d}}{\mathrm{d} t} q_{A_{t}}\right|_{t=t_{0}}=q_{\dot{A}_{t_{0}}} .
$$

(3) If 1 does not belong to the spectrum of $A$, then the function $\mathcal{T} \ni X \mapsto F(X)=\operatorname{det}(I-X)$ is differentiable at $X=A$ and its derivative is given by

$$
\left(\mathrm{D}_{A} F\right)(X)=-F(A) \operatorname{tr}\left((I-A)^{-1} X\right) .
$$

Proof.

Part (1) By Eq. (4), the function $x \mapsto \Phi_{y}(x)=(y, x)$ belongs to $L^{2}\left(\mathfrak{X}, \mathrm{d} \omega_{D}\right)$ for $y \in \mathfrak{X}_{l}^{*}$. Moreover, Fubini's theorem yields the estimate

$$
\left\|\Phi_{y}\right\|_{2}^{2}=\sum_{i, j \in \Gamma} y_{i} y_{j} \int x_{i} x_{j} \mathrm{~d} \omega_{D}(x)=\sum_{i, j \in \Gamma} D_{i j} y_{i} y_{j}=(y, D y) \leq\|D\|\|y\|^{2},
$$

which implies that the linear map $y \mapsto \Phi_{y}$ has a unique extension $\Phi: \ell_{\mathbb{R}}^{2}(\Gamma) \rightarrow L^{2}\left(\mathfrak{X}, \mathrm{d} \omega_{D}\right)$, such that $\|\Phi\| \leq\|D\|^{1 / 2}$.

\footnotetext{
${ }^{3}$ In the case of harmonic crystal considered in this section, $j$ is a discrete Laplacian and the absolute values of the entries of the scattering matrix of the pair $\left(j, j_{0}\right)$ are either 0 's or 1's. For this reason the formula (32) for $e(\alpha)$ has a particularly simple form.
} 
A self-adjoint $A \in \mathcal{T}$ has a spectral representation $A=\sum_{k} a_{k} \varphi_{k}\left(\varphi_{k}, \cdot\right)$, where the $a_{k}$ are the eigenvalues of $A$ and the corresponding eigenvectors $\varphi_{k}$ form an orthonormal basis of $\ell_{\mathbb{R}}^{2}(\Gamma)$. It follows that $q_{A}(x)=$ $\sum_{k} a_{k} \Phi_{\varphi_{k}}(x)^{2}$ from which we conclude that $q_{A}$ extends to an element of $L^{1}\left(\mathfrak{X}, \mathrm{d} \omega_{D}\right)$ with

$$
\left\|q_{A}\right\|_{1} \leq \sum_{k}\left|a_{k}\right|\left\|\Phi_{\varphi_{k}}\right\|_{2}^{2} \leq \sum_{k}\left|a_{k}\right|\|D\|=\|D\|\|A\|_{1} .
$$

The last equality in Eq. (36) yields

$$
\int q_{A}(x) \mathrm{d} \omega_{D}(x)=\sum_{k} a_{k}\left\|\Phi_{\varphi_{k}}\right\|_{2}^{2}=\sum_{k} a_{k}\left(\varphi_{k}, D \varphi_{k}\right)=\operatorname{tr}(A D),
$$

which proves Identity (34).

Part (2) It follows from Part (1) that the linear map $\mathcal{T} \ni A \mapsto q_{A} \in L^{1}\left(\mathfrak{X}, \mathrm{d} \omega_{D}\right)$ is bounded and hence $C^{1}$.

Part (3) Using a well known property of the determinant (see Theorem 3.5 in [Si]), we can write

$$
\begin{aligned}
F(A+X)=\operatorname{det}(I-(A+X)) & =\operatorname{det}\left((I-A)\left(I-(I-A)^{-1} X\right)\right. \\
& =\operatorname{det}(I-A) \operatorname{det}\left(I-(I-A)^{-1} X\right) \\
& =F(A) \operatorname{det}\left(I-(I-A)^{-1} X\right) .
\end{aligned}
$$

To evaluate the second factor on the right-hand side of this identity, we apply the formula

$$
\operatorname{det}(I+Q)=1+\sum_{k=1}^{\infty} \operatorname{tr}\left(Q^{\wedge k}\right)
$$

where $Q^{\wedge k}$ denotes the $k$-th antisymmetric tensor power of $Q$ (see [Si]). Since $\left\|Q^{\wedge k}\right\|_{1} \leq(k !)^{-1}\|Q\|_{1}^{k}$, one has the estimate

$$
|\operatorname{det}(I+Q)-1-\operatorname{tr}(Q)| \leq \mathrm{e}^{\|Q\|_{1}}-1-\|Q\|_{1} \leq \frac{\mathrm{e}^{\|Q\|_{1}}}{2}\|Q\|_{1}^{2} .
$$

It follows that

$$
\operatorname{det}\left(I-(I-A)^{-1} X\right)=1-\operatorname{tr}\left((I-A)^{-1} X\right)+\mathcal{O}\left(\|X\|_{1}^{2}\right),
$$

as $X \rightarrow 0$ in $\mathcal{T}$. Thus, we can conclude that

$$
F(A+X)-F(A)=-F(A) \operatorname{tr}\left((I-A)^{-1} X\right)+\mathcal{O}\left(\|X\|_{1}^{2}\right),
$$

and the result follows

\subsection{Proof of Proposition 2.1}

Part (1) Up to the constant $\operatorname{tr}(D \varsigma)$ (which is well defined since $\varsigma \in \mathcal{T}$ ), $\sigma$ is given by the quadratic form $q_{\varsigma}$ which is in $L^{1}(\mathfrak{X}, \mathrm{d} \omega)$ by Lemma $4.1(1)$. For $x \in \mathfrak{X}_{l}$, i.e., $\omega$-a.e. $x \in \mathfrak{X}$, one has

$$
\sigma_{t}(x)-\sigma_{s}(x)=\frac{1}{2}\left(x,\left(\mathrm{e}^{t \mathcal{L}^{*}} \varsigma^{t \mathcal{L}}-\mathrm{e}^{s \mathcal{L}^{*}} \varsigma^{s \mathcal{L}}\right) x\right),
$$

whence, setting $\varsigma_{t}=\mathrm{e}^{t \mathcal{L}^{*}} \varsigma \mathrm{e}^{t \mathcal{L}}$ and applying again Lemma 4.1 (1), it follows that

$$
\left\|\sigma_{t}-\sigma_{s}\right\|_{L^{1}(\mathfrak{X}, \mathrm{d} \omega)} \leq \frac{1}{2}\|D\|\left\|\varsigma_{t}-\varsigma_{s}\right\|_{1} .
$$

Thus, it suffices to show that the function $t \mapsto \varsigma_{t} \in \mathcal{T}$ is continuous. This immediately follows from the norm continuity of the group $\mathrm{e}^{t \mathcal{L}}$, the fact that $\varsigma \in \mathcal{T}$, and the well-known trace inequality $\|A B\|_{1} \leq$ $\|A\|\|B\|_{1}$. We note, in particular, that

$$
\left\|\sigma_{t}\right\|_{L^{1}(\mathfrak{X}, \mathrm{d} \omega)} \leq\|D\|\left(1+\left\|\mathrm{e}^{t \mathcal{L}}\right\|^{2}\right)\|\varsigma\|_{1} \quad \text { for } t \in \mathbb{R} .
$$


Part (2) From Eq. (5), we deduce that

$$
\ell_{\omega_{t} \mid \omega}=\frac{1}{2} \log \operatorname{det}\left(I+D T_{t}\right)-\frac{1}{2} q_{T_{t}} .
$$

Now note that $T_{t}=D_{t}^{-1}-D^{-1}$ satisfies the cocycle relation

$$
T_{t+s}=T_{t}+\mathrm{e}^{-t \mathcal{L}^{*}} T_{s} \mathrm{e}^{-t \mathcal{L}} .
$$

It thus follows from Assumption (G1) that the function $t \mapsto T_{t} \in \mathcal{T}$ is everywhere differentiable and that its derivative is given by

$$
\dot{T}_{t}=-2 \varsigma_{-t} .
$$

Lemma 4.1 (3) and the chain rule imply that the first term on the right-hand side of (37) is a differentiable function of $t$. Using Eq. (35), an elementary calculation shows that

$$
\left.\frac{1}{2} \frac{\mathrm{d}}{\mathrm{d} t} \log \operatorname{det}\left(I+D T_{t}\right)\right|_{t=0}=-\operatorname{tr}(D \varsigma)
$$

Applying Lemma 4.1 (2) to the second term on the right-hand side of Eq. (37), one further gets

$$
-\frac{1}{2} \frac{\mathrm{d}}{\mathrm{d} t} q_{T_{t}}=q_{\varsigma_{-t}}=q_{\varsigma} \circ \phi^{-t} .
$$

Summing up, we have shown that

$$
\frac{\mathrm{d}}{\mathrm{d} t} \ell_{\omega_{t} \mid \omega}=\sigma_{-t}, \quad t \in \mathbb{R} .
$$

Since the function $t \mapsto \sigma_{-t} \in L^{1}(\mathfrak{X}, \mathrm{d} \omega)$ is continuous by Lemma 4.1 (1), and $\ell_{\omega \mid \omega}=0$, we can use Riemann's integral to write

$$
\ell_{\omega_{t} \mid \omega}=\int_{0}^{t} \sigma_{-s} \mathrm{~d} s
$$

The fact that, for $\omega$-almost every $x \in \mathfrak{X}$, one has

$$
\ell_{\omega_{t} \mid \omega}(x)=\int_{0}^{t} \sigma_{-s}(x) \mathrm{d} s
$$

follows from Theorem 3.4.2 in [HP].

Part (3) From the cocycle relation

$$
\ell_{\omega_{t+s} \mid \omega}=\ell_{\omega_{t} \mid \omega}+\ell_{\omega_{s} \mid \omega} \circ \phi^{-t}
$$

we infer

$$
\xi_{s}=\frac{1}{s}\left(\mathrm{e}^{\ell_{\omega_{t+s} \mid \omega}}-\mathrm{e}^{\ell_{\omega_{t} \mid \omega}}\right)-\sigma_{-t} \mathrm{e}^{\ell_{\omega_{t} \mid \omega}}=\frac{1}{s}\left(\mathrm{e}^{\ell_{\omega_{s} \mid \omega}}-1-s \sigma\right) \circ \phi^{-t} \frac{\mathrm{d} \omega_{t}}{\mathrm{~d} \omega},
$$

and hence

$$
\int_{\mathfrak{X}}\left|\xi_{s}\right| \mathrm{d} \omega=\frac{1}{|s|} \int_{\mathfrak{X}}\left|\mathrm{e}^{\ell_{\omega_{s} \mid \omega}}-1-s \sigma\right| \mathrm{d} \omega \leq \frac{1}{|s|} \int_{\mathfrak{X}}\left|\mathrm{e}^{\ell_{\omega_{s} \mid \omega}}-1-\ell_{\omega_{s} \mid \omega}\right| \mathrm{d} \omega+\frac{1}{|s|} \int_{\mathfrak{X}}\left|\ell_{\omega_{s} \mid \omega}-s \sigma\right| \mathrm{d} \omega .
$$

To prove that Relation (9) holds in $L^{1}(\mathfrak{X}, \mathrm{d} \omega)$, it suffices to show that both terms on the right-hand side of this inequality vanish in the limit $s \rightarrow 0$.

To estimate the first term we note that the inequality $\mathrm{e}^{\ell}-1-\ell \geq 0$ (which holds for $\ell \in \mathbb{R}$ ) combined with Eq. (34) and (37) implies

$$
\begin{aligned}
\frac{1}{|s|} \int_{\mathfrak{X}}\left|\mathrm{e}^{\ell_{\omega_{s} \mid \omega}}-1-\ell_{\omega_{s} \mid \omega}\right| \mathrm{d} \omega & =\frac{1}{|s|}\left(\omega\left(\mathrm{e}^{\ell_{\omega_{s} \mid \omega}}\right)-1-\int_{\mathfrak{X}} \ell_{\omega_{s} \mid \omega} \mathrm{d} \omega\right) \\
& =\frac{1}{2}\left|\frac{1}{s}\left(\operatorname{tr}\left(D T_{s}\right)-\log \operatorname{det}\left(I+D T_{s}\right)\right)\right| .
\end{aligned}
$$


By Assumption (G1), the map $s \mapsto T_{s}$ is differentiable in $\mathcal{T}$ at $s=0$. Since $T_{0}=0$, we can write

$$
\lim _{s \rightarrow 0} \frac{1}{|s|} \int_{\mathfrak{X}}\left|\mathrm{e}^{\ell_{\omega_{s} \mid \omega}}-1-\ell_{\omega_{s} \mid \omega}\right| \mathrm{d} \omega=\frac{1}{2}\left|\frac{\mathrm{d}}{\mathrm{d} s}\left(\operatorname{tr}\left(D T_{s}\right)-\log \operatorname{det}\left(I+D T_{s}\right)\right)\right|_{s=0} \mid .
$$

Using Lemma 4.1 (3) and the chain rule, we get

$$
\left.\frac{\mathrm{d}}{\mathrm{d} s}\left(\operatorname{tr}\left(D T_{s}\right)-\log \operatorname{det}\left(I+D T_{s}\right)\right)\right|_{s=0}=\operatorname{tr}\left(D \dot{T}_{0}\right)-\operatorname{tr}\left(D \dot{T}_{0}\right)=0 .
$$

To deal with the second term, we use Eq. (40), Fubini's theorem and Lemma 4.1 (1) to write

$$
\begin{aligned}
\frac{1}{|s|} \int_{\mathfrak{X}}\left|\ell_{\omega_{s} \mid \omega}-s \sigma\right| \mathrm{d} \omega & =\int_{\mathfrak{X}}\left|\int_{0}^{1}\left(\sigma_{-s u}-\sigma\right) \mathrm{d} u\right| \leq \int_{0}^{1} \int_{\mathfrak{X}}\left|q_{\varsigma_{-s u}-\varsigma}\right| \mathrm{d} \omega \mathrm{d} u \\
& \leq\|D\| \int_{0}^{1}\left\|\varsigma_{-s u}-\varsigma\right\|_{1} \mathrm{~d} u,
\end{aligned}
$$

and since the map $s \mapsto \varsigma_{s}$ is continuous in $\mathcal{T}$, the dominated convergence theorem yields

$$
\lim _{s \rightarrow 0} \int_{0}^{1}\left\|\varsigma_{-s u}-\varsigma\right\|_{1} \mathrm{~d} u=0 .
$$

Part (4) Relation (7) implies that

$$
\omega_{t}(\sigma)=\omega\left(\sigma_{t}\right)=\int_{\mathfrak{X}} q_{\varsigma_{t}} \mathrm{~d} \omega-\operatorname{tr}(D \varsigma)
$$

and formula (34) yields

$$
\omega_{t}(\sigma)=\operatorname{tr}\left(D\left(\varsigma_{t}-\varsigma\right)\right)=\operatorname{tr}\left(\varsigma\left(D_{t}-D\right)\right) .
$$

Part (5) Starting from Definition (2) and using the cocycle relation (42), we obtain

$$
\operatorname{Ent}\left(\omega_{t} \mid \omega\right)=-\int_{\mathfrak{X}} \ell_{\omega_{t} \mid \omega} \mathrm{d} \omega_{t}=\int_{\mathfrak{X}} \ell_{\omega_{-t} \mid \omega} \mathrm{d} \omega .
$$

Eq. (41) and Fubini's theorem further yield

$$
\operatorname{Ent}\left(\omega_{t} \mid \omega\right)=\int_{\mathfrak{X}} \int_{0}^{-t} \sigma_{-s} \mathrm{~d} s \mathrm{~d} \omega=-\int_{\mathfrak{X}} \int_{0}^{t} \sigma_{s} \mathrm{~d} s \mathrm{~d} \omega=-\int_{0}^{t} \omega_{s}(\sigma) \mathrm{d} s .
$$

\subsection{Proof of Proposition 2.2}

Part (1) We have to show that $\omega_{+}$, the Gaussian measure of covariance $D_{+}$, is the weak limit of the net $\left\{\omega_{t}\right\}_{t>0}$. Since the cylinders form a convergence determining class for Borel measures on $\mathfrak{X}$ (see Example 2.4 in [Bill]), it suffices to show that $\lim _{t \rightarrow \infty} \omega_{t}\left(C_{I}(B)\right)=\omega_{+}\left(C_{I}(B)\right)$ holds for any finite subset $I \subset \Gamma$ and any Borel set $B \subset \mathbb{R}^{I}$. By Hypotheses (G2)-(G3), one has $\lim _{t \rightarrow \infty} D_{t, I}=D_{+, I}$ and

$$
\mathrm{e}^{-\frac{1}{2}\left(x, D_{t, I}^{-1} x\right)} \leq \mathrm{e}^{-\frac{\|x\|^{2}}{2 M}}
$$

for all $x \in \mathbb{R}^{I}$. It follows that $\lim _{t \rightarrow \infty} D_{t, I}^{-1}=D_{+, I}^{-1}$ as well as $\lim _{t \rightarrow \infty} \operatorname{det}\left(2 \pi D_{t, I}\right)=\operatorname{det}\left(2 \pi D_{+, I}\right)$ so that

$$
\lim _{t \rightarrow \infty} \frac{1}{\sqrt{\operatorname{det}\left(2 \pi D_{t, I}\right)}} \int_{B} \mathrm{e}^{-\frac{1}{2}\left(x, D_{t, I}^{-1} x\right)} \mathrm{d} x=\frac{1}{\sqrt{\operatorname{det}\left(2 \pi D_{+, I}\right)}} \int_{B} \mathrm{e}^{-\frac{1}{2}\left(x, D_{+, I}^{-1} x\right)} \mathrm{d} x,
$$

holds by the dominated convergence theorem. The same argument applies to $\omega_{-}$.

Part (2) Follows directly from Lemma 4.1 (1) and Proposition 2.1 (4). 


\subsection{Proof of Proposition 2.3}

Part (1) Let us note that $\alpha \in J_{t}$ if and only if

$$
D^{-1}+\alpha\left(\mathrm{e}^{-t \mathcal{L}^{*}} D^{-1} \mathrm{e}^{-t \mathcal{L}}-D^{-1}\right)>0 .
$$

It follows that $J_{t}$ is open. For $\theta \in[0,1]$, we can write

$$
D^{-1}+\theta \alpha\left(\mathrm{e}^{-t \mathcal{L}^{*}} D^{-1} \mathrm{e}^{-t \mathcal{L}}-D^{-1}\right)=\theta\left(D^{-1}+\alpha\left(\mathrm{e}^{-t \mathcal{L}^{*}} D^{-1} \mathrm{e}^{-t \mathcal{L}}-D^{-1}\right)\right)+(1-\theta) D^{-1},
$$

whence $\alpha \in J_{t} \Rightarrow \theta \alpha \in J_{t}$ and we can conclude that $J_{t}$ is an interval. Multiplying (43) by $\vartheta$ from the left and the right and using the relations $\vartheta=\vartheta^{*}=\vartheta^{-1}$, we obtain

$$
D^{-1}+\alpha\left(\mathrm{e}^{t \mathcal{L}^{*}} D^{-1} \mathrm{e}^{t \mathcal{L}}-D^{-1}\right)>0,
$$

whence we see that $\alpha \in J_{-t}$. By symmetry, we conclude that $J_{-t}=J_{t}$. Furthermore, multiplying (44) by $\mathrm{e}^{-t \mathcal{L}^{*}}$ and $\mathrm{e}^{-t \mathcal{L}}$ from the left and the right, respectively, we obtain

$$
\alpha D^{-1}+(1-\alpha) \mathrm{e}^{-t \mathcal{L}^{*}} D^{-1} \mathrm{e}^{-t \mathcal{L}}>0 .
$$

It follows that $1-\alpha \in J_{t}$, and by symmetry, we conclude that $\alpha \in J_{t}$ if and only if $1-\alpha \in J_{t}$. Thus, $J_{t}$ is an open interval symmetric around $\alpha=\frac{1}{2}$.

Part (2) For any bounded operator $C>0$ on $\ell_{\mathbb{R}}^{2}(\Gamma)$ and for any $\alpha, t \in \mathbb{R}$ such that $C^{-1}+\alpha T_{t}>0$, formulas (5) and (37) allow us to write

$$
\mathrm{e}^{\alpha \ell_{\omega_{t} \mid \omega}} \mathrm{d} \omega_{C}=\sqrt{\frac{\left(\operatorname{det}\left(I+D T_{t}\right)\right)^{\alpha}}{\operatorname{det}\left(I+\alpha C T_{t}\right)}} \mathrm{d} \omega_{\left(C^{-1}+\alpha T_{t}\right)^{-1}} .
$$

By definition $D^{-1}+\alpha T_{t}>0$ for $\alpha \in\left(-\delta_{t}, 1+\delta_{t}\right)$. Taking $C=D$ in (45) and integrating over $\mathfrak{X}$, one easily checks that

$$
e_{t}(\alpha)=\frac{\alpha}{2} \log \operatorname{det}\left(I+D T_{t}\right)-\frac{1}{2} \log \operatorname{det}\left(I+\alpha D T_{t}\right)
$$

for all $t \in \mathbb{R}$ and $\alpha \in\left(-\delta_{t}, 1+\delta_{t}\right)$. The first term on the right-hand side of this identity is linear in $\alpha$ and hence entire analytic. ${ }^{4}$ The determinant in the second term is also an entire function of $\alpha$, and its logarithm is analytic on the set where the operator $I+\alpha D T_{t}$ is invertible; see Section IV.1 in [GK]. Writing $I+\alpha D T_{t}=D\left(D^{-1}+\alpha T_{t}\right)$, we see that $I+\alpha D T_{t}$ is invertible for $\alpha \in J_{t}$. Furthermore, since

$$
I+\alpha D T_{t}=\alpha D^{1 / 2}\left(\alpha^{-1} I+D^{1 / 2} T_{t} D^{1 / 2}\right) D^{-1 / 2},
$$

and the operator $D^{1 / 2} T_{t} D^{1 / 2}$ is self-adjoint, we conclude that $I+\alpha D T_{t}$ is invertible for $\alpha \in \mathbb{C} \backslash \mathbb{R}$. Hence, the function $e_{t}(\alpha)$ is analytic in the cut plane $\mathbb{C}_{+} \cup \mathbb{C}_{-} \cup J_{t}$. Its convexity is a well-known property of Rényi's relative entropy and follows from Hölder's inequality applied to Eq. (12), and relations (13) are easy to check by a direct computation.

It remains to prove that $e_{t}(\alpha)=+\infty$ for $\alpha \notin J_{t}$. To this end, we first note that the spectrum of $D^{-1}$ is contained in the interval $\left[M^{-1}, m^{-1}\right]$ and that the operator $\alpha T_{t}$ is compact. By the Weyl theorem on essential spectrum, it follows that the intersection of the spectrum of the self-adjoint operator $D^{-1}+\alpha T_{t}$ with the complement of $\left[M^{-1}, m^{-1}\right]$ consists of isolated eigenvalues. Thus, if $\alpha \notin J_{t}$, then there are finitely many orthonormal vectors $\left\{\varphi_{j}\right\}$, numbers $\lambda_{j} \geq 0$, and an operator $B \geq c I$ with $c>0$ such that

$$
D^{-1}+\alpha T_{t}=-\sum_{j=1}^{n} \lambda_{j}\left(\varphi_{j}, \cdot\right) \varphi_{j}+B
$$

\footnotetext{
${ }^{4}$ We shall see in the proof of Theorem 2.4 that it is in fact identically equal to zero.
} 
It follows that

$$
\omega\left(\mathrm{e}^{\alpha \ell_{\omega_{t} \mid \omega}}\right)=\left(\operatorname{det}\left(I+D T_{t}\right)\right)^{\alpha / 2} \int_{\mathfrak{X}} \exp \left\{\frac{1}{2} \sum_{j=1}^{n} \lambda_{j}\left|\left(\varphi_{j}, x\right)\right|^{2}\right\} \mathrm{e}^{-(x, B x) / 2} \omega(\mathrm{d} x) .
$$

Since $B-D^{-1} \in \mathcal{T}$ and $D^{-1}+B>0$, we conclude from (5) that $\mathrm{e}^{-(x, B x) / 2} \omega(\mathrm{d} x)$ coincides, up to a numerical factor $C>0$, with a centered Gaussian measure whose covariance operator is equal to $D^{\prime}:=\left(D^{-1}+B\right)^{-1}$. Hence, we can rewrite (47) in the form

$$
\omega\left(\mathrm{e}^{\alpha \ell_{\omega_{t} \mid \omega}}\right)=C \int_{\mathfrak{X}} \exp \left\{\frac{1}{2} \sum_{j=1}^{n} \lambda_{j}\left|\left(\varphi_{j}, x\right)\right|^{2}\right\} \omega_{D^{\prime}}(\mathrm{d} x) .
$$

Since the support of $\omega_{D^{\prime}}$ coincides with the entire space, this integral is infinite.

Part (3) Using the cocycle relation (42), we can write ${ }^{5}$

$$
\begin{aligned}
e_{t}(1-\alpha) & =\log \omega\left(\mathrm{e}^{\ell_{\omega_{t} \mid \omega}} \mathrm{e}^{-\alpha \ell_{\omega_{t} \mid \omega}}\right)=\log \omega_{t}\left(\mathrm{e}^{-\alpha \ell_{\omega_{t} \mid \omega}}\right) \\
& =\log \omega\left(\mathrm{e}^{-\alpha \ell_{\omega_{t} \mid \omega} \circ \phi^{t}}\right)=\log \omega\left(\mathrm{e}^{\alpha \ell_{\omega_{-t} \mid \omega}}\right)=e_{-t}(\alpha) .
\end{aligned}
$$

Now note that, by (G4), the measure $\omega$ is invariant under $\vartheta$, whence we conclude that $\omega_{-t}=\omega_{t} \circ \vartheta$ and $\ell_{\omega_{t} \mid \omega} \circ \vartheta=\ell_{\omega_{-t} \mid \omega}$. It follows that $e_{-t}(\alpha)=e_{t}(\alpha)$. Combining this with the above relation, we obtain the Evans-Searles symmetry.

\subsection{Proof of Theorem 2.4}

Part (1) We first prove the existence of limit (14). Let us set

$$
D_{t}(\alpha)=\left((1-\alpha) D^{-1}+\alpha D_{t}^{-1}\right)^{-1}
$$

and recall that $e_{t}(\alpha)$ can be written in the form (46). Using Relations (35), (39), Lemma 4.1 (3) and the chain rule we obtain

$$
\frac{\mathrm{d}}{\mathrm{d} t} \log \operatorname{det}\left(I+\alpha D T_{t}\right)=\operatorname{tr}\left(\left(I+\alpha D T_{t}\right)^{-1} \alpha D \dot{T}_{t}\right)=-2 \alpha \operatorname{tr}\left(D_{t}(\alpha) \varsigma_{-t}\right)=-2 \alpha \operatorname{tr}\left(D_{-t}(1-\alpha) \varsigma\right) .
$$

In particular, for $\alpha=1$ the derivative is equal to zero for any $t \in \mathbb{R}$, whence we conclude that the first term in (46) is identically equal to zero. Let us now fix $\alpha \in \underline{J}$ and choose $t_{0}>0$ so large that $\alpha \in J_{t}$ for $t \geq t_{0}$. It follows from (46) and (49) that

$$
\frac{1}{t} e_{t}(\alpha)=\frac{1}{t} e_{t_{0}}(\alpha)-\frac{2 \alpha}{t} \int_{t_{0}}^{t} \operatorname{tr}\left(D_{-s}(1-\alpha) \varsigma\right) \mathrm{d} s
$$

By Assumption (G3)

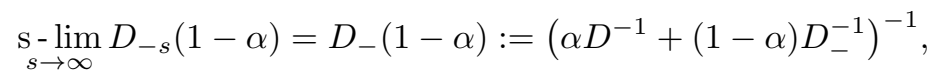

and since $\varsigma$ is trace class, it follows that

$$
\lim _{s \rightarrow \infty} \operatorname{tr}\left(D_{s}(1-\alpha) \varsigma\right)=\operatorname{tr}\left(D_{-}(1-\alpha) \varsigma\right) .
$$

Combining this with (50), we conclude that for $\alpha \in \underline{J}$,

$$
\lim _{t \rightarrow+\infty} \frac{1}{t} e_{t}(\alpha)=-2 \alpha \operatorname{tr}\left(D_{-}(1-\alpha) \varsigma\right) .
$$

\footnotetext{
${ }^{5}$ Note that this computation does not use $(\mathrm{G} 4)$.
} 
Once the existence of limit is known, we can easily obtain the required properties of $e(\alpha)$. The convexity of $e(\alpha)$ and the first and last relations in (15) follow immediately from the corresponding properties of $e_{t}(\alpha)$. Furthermore, it follows from (40) and the invariance of $\omega$ under $\vartheta$ that

$$
e_{t}^{\prime}(0)=\int_{\mathfrak{X}} \ell_{\omega_{t} \mid \omega}(x) \omega(\mathrm{d} x)=\int_{\mathfrak{X}} \int_{0}^{t} \sigma_{-s}(x) \mathrm{d} s \omega(\mathrm{d} x)=-\int_{\mathfrak{X}} \int_{0}^{t} \sigma_{s}(x) \mathrm{d} s \omega(\mathrm{d} x) .
$$

In view of Part (2), the limit $e(\alpha)$ is analytic on its domain of definition. By Theorem 25.7 in [Rock],

$$
\lim _{t \rightarrow \infty} \frac{1}{t} e_{t}^{\prime}(\alpha)=e^{\prime}(\alpha)
$$

for $\alpha \in \underline{J}$. Using Fubini's theorem and Part (2) of Proposition 2.2, we derive

$$
e^{\prime}(0)=\lim _{t \rightarrow \infty} \frac{1}{t} e_{t}^{\prime}(0)=-\lim _{t \rightarrow \infty} \frac{1}{t} \int_{0}^{t} \omega\left(\sigma_{s}\right) \mathrm{d} s=-\omega_{+}(\sigma)=-\operatorname{tr}\left(\varsigma D_{+}\right) .
$$

The third relation in (15) now follows from the fourth one.

Part (2) The analyticity of $e(\alpha)$ follows from Relation (51). We now prove (16).

Let $\mu$ be the spectral measure of $Q$ for the linear functional induced by the trace class operator $D_{-}^{1 / 2}{ }_{\varsigma} D_{-}^{1 / 2}$. In other words, $\mu$ is the signed Borel measure such that

$$
\int f(q) \mu(\mathrm{d} q)=\operatorname{tr}\left(f(Q) D_{-}^{1 / 2}{ }_{\varsigma} D_{-}^{1 / 2}\right)
$$

for any bounded continuous function $f: \mathbb{R} \rightarrow \mathbb{C}$. By Eq. (20), the measure $\mu$ has its support in the interval $\left[-\bar{\delta}^{-1},(1+\bar{\delta})^{-1}\right]$. One easily checks that

$$
f \mapsto \int f\left(q^{-1}\right) q^{-1} \mu(\mathrm{d} q),
$$

defines a continuous linear functional on the Fréchet space $C_{0}(\mathbb{R})$ of compactly supported continuous functions $f: \mathbb{R} \rightarrow \mathbb{C}$. By the Riesz representation theorem (see Chapter 2 in [Rud]), it follows that there exists a signed Borel measure $\nu$, with support on $(-\infty,-\bar{\delta}] \cup[1+\bar{\delta}, \infty)$, such that

$$
\int f(r) \nu(\mathrm{d} r)=\int f\left(q^{-1}\right) q^{-1} \mu(\mathrm{d} q)
$$

A standard argument based on the monotone class technique shows that (53) remains valid for any bounded measurable function $f$. Decomposing the measures $\mu$ and $\nu$ into their positive and negative parts, we easily deduce from (53) that

$$
\int f(r)|\nu|(\mathrm{d} r)=\int f\left(q^{-1}\right)|q|^{-1}|\mu|(\mathrm{d} q)
$$

for all bounded continuous $f$. In particular, taking $f(r)=\frac{1}{r}$ outside a small neighborhood of zero and using (52), we derive

$$
\int \frac{|\nu|(\mathrm{d} r)}{|r|}=\int|\mu|(\mathrm{d} q) \leq\left\|D_{-}^{1 / 2}{ }_{\varsigma} D_{-}^{1 / 2}\right\|_{1}<\infty .
$$

Recalling relation (22) (which will be established below) and using (53) with $f(r)=-\log \left(1-\alpha r^{-1}\right)$ on the support of $\nu$, we obtain

$$
\begin{aligned}
e(\alpha) & =-\alpha \operatorname{tr}\left(g(\alpha Q) D_{-}^{1 / 2}{ }_{\varsigma} D_{-}^{1 / 2}\right)=-\int \alpha g(\alpha q) \mu(\mathrm{d} q) \\
& =-\int q^{-1} \log (1-\alpha q) \mu(\mathrm{d} q)=-\int \log \left(1-\alpha r^{-1}\right) \nu(\mathrm{d} r) .
\end{aligned}
$$

This relation coincides with (16). 
To prove the uniqueness, let $\nu_{1}, \nu_{2}$ be two signed Borel measures with support in $\mathbb{R} \backslash \underline{J}$, satisfying $\int|r|^{-1}\left|\nu_{k}\right|(\mathrm{d} r)<\infty, k=1,2$, and such that

$$
\int \log \left(1-\alpha r^{-1}\right) \nu_{1}(\mathrm{~d} r)=\int \log \left(1-\alpha r^{-1}\right) \nu_{2}(\mathrm{~d} r)
$$

for $\alpha \in \underline{J}$. Differentiating, we derive that

$$
\int \frac{\mathrm{d} \nu_{1}(r)}{r-\alpha}=\int \frac{\mathrm{d} \nu_{2}(r)}{r-\alpha}
$$

for $\alpha \in \underline{J}$. By analytic continuation (54) holds for all $\alpha \in \mathbb{C}_{+} \cup \mathbb{C}_{-}$. Since the linear span of the set of functions $\left\{(r-\alpha)^{-1} \mid \alpha \in \mathbb{C}_{+} \cup \mathbb{C}_{-}\right\}$is dense in $C_{0}(\mathbb{R})$, (54) yields that for any $f \in C_{0}(\mathbb{R})$, $\int f \mathrm{~d} \nu_{1}=\int f \mathrm{~d} \nu_{2}$. Hence $\nu_{1}=\nu_{2}$.

Part (3) The fact that $I$ is a convex function taking values in $[0,+\infty]$ follows immediately from the definition. The relation $e^{\prime}(0)=\omega_{-}(\sigma)=-\omega_{+}(\sigma)$ and the regularity of $e$ imply that $I$ vanishes only at $s=\omega_{+}(\sigma)$. The validity of (17) is a straightforward consequence of the last relation in (15). Let us prove (18).

Consider the following family of random variables $\left\{\Sigma_{t}\right\}_{t \in[0, \infty)}$ defined on the probability space $(\mathfrak{X}, \mathcal{F}, \omega)$

$$
\Sigma_{t}=\frac{1}{t} \int_{0}^{t} \sigma_{s} \mathrm{~d} s
$$

By Proposition 2.1 (2) and the symmetry relations $\omega=\omega \circ \vartheta$ and $\sigma \circ \vartheta=-\sigma$, we have

$$
e_{t}(\alpha)=\log \omega\left(\mathrm{e}^{\alpha \ell_{\omega_{t}} \mid \omega}\right)=\log \omega\left(\mathrm{e}^{\alpha \int_{0}^{t} \sigma_{-s} \mathrm{~d} s}\right)=\log \omega\left(\mathrm{e}^{-\alpha \int_{0}^{t} \sigma_{s} \mathrm{~d} s}\right)=\log \omega\left(\mathrm{e}^{-\alpha t \Sigma_{t}}\right),
$$

so that $e_{t}(-\alpha)$ is the cumulant generating function of the family $\left\{\Sigma_{t}\right\}_{t \in[0, \infty)}$. Applying a local version of the Gärtner-Ellis theorem (see Theorem 4.65 in [JOPP]), we conclude that (18) holds with

$$
\varepsilon=\min \left(-\omega_{+}(\sigma)-\partial^{+} e(-\underline{\delta}),-\omega_{+}(\sigma)+\partial^{-} e(1+\underline{\delta})\right)=\min \left(e^{\prime}(0)-\partial^{+} e(-\underline{\delta}), \partial^{-} e(1+\underline{\delta})-e^{\prime}(1)\right)
$$

where $\partial^{ \pm} e(\alpha)$ denotes the right/left derivative of $e(\alpha)$. The fact that $\varepsilon>0$ follows from the convexity and analyticity of $e(\alpha)$.

Part (4) As was shown above, $e_{t}(-\alpha)$ is the cumulant generating function of $\left\{\Sigma_{t}\right\}$. Therefore, by Bryc's lemma (see [Br] or Section 4.8.4 in [JOPP]), the CLT will be established if we prove that $e_{t}(\alpha)$ extends analytically to a disc $\mathcal{D}_{\varepsilon}=\{\alpha \in \mathbb{C}|| \alpha \mid<\varepsilon\}$ and satisfies the estimate

$$
\sup _{t \geq t_{0}, \alpha \in \mathcal{D}_{\varepsilon}} \frac{1}{t}\left|e_{t}(\alpha)\right|<\infty
$$

for some $t_{0}>0$. The analyticity was established in Part (2) of Proposition 2.3. Using the representation (50), one easily sees that in order to prove (55) it suffices to show that

$$
\sup _{t \in \mathbb{R},|1-\alpha|<\varepsilon}\left\|D_{t}(\alpha)\right\|<\infty .
$$

An elementary analysis shows that Assumption (G2) implies the lower bound

$$
(1-\alpha) D_{s}^{-1}+\alpha D_{t}^{-1} \geq \frac{2}{M} \frac{M-m}{M+m}\left(\delta+\frac{1}{2}-\left|\alpha-\frac{1}{2}\right|\right),
$$

for $t, s \in \mathbb{R}$ and $\alpha \in[-\delta, 1+\delta]$. Since for $z \in \mathbb{C}$

$$
\operatorname{Re}\left((1-z) D_{s}^{-1}+z D_{t}^{-1}\right)=(1-\operatorname{Re} z) D_{s}^{-1}+\operatorname{Re} z D_{t}^{-1},
$$

we have the upper bound

$$
\left\|\left((1-z) D_{s}^{-1}+z D_{t}^{-1}\right)^{-1}\right\| \leq \frac{M}{2} \frac{M+m}{M-m}\left(\delta+\frac{1}{2}-\left|\operatorname{Re} z-\frac{1}{2}\right|\right)^{-1}
$$


for $s, t \in \mathbb{R}$ and $z$ in the strip $\{z \in \mathbb{C} \mid \operatorname{Re} z \in(-\delta, 1+\delta)\}$. Thus, the required estimate (56) holds provided $\epsilon<\delta$.

Part (5) We first note that the differentiability of $e(\alpha)$ at zero and a local version of Theorems II.6.3 in [El] (which holds with identical proof) implies that, for any $\varepsilon>0$ and any integer $n \geq 1$,

$$
\omega\left(\left\{x \in \mathfrak{X}|| \Sigma_{n}-\omega_{+}(\sigma) \mid \geq \varepsilon\right\}\right) \leq \mathrm{e}^{-a(\varepsilon) n},
$$

where $a(\varepsilon)>0$ does not depend on $n$. By Theorems II.6.4 in [El], it follows that

$$
\lim _{n \rightarrow \infty} \frac{1}{n} \int_{0}^{n} \sigma_{s}(x) \mathrm{d} s=\omega_{+}(\sigma)
$$

for $\omega$-a.e. $x \in \mathfrak{X}$. Suppose now we have shown the following inequality for some $r<1$

$$
\sup _{0 \leq t \leq 1}\left|\int_{n}^{n+t} \sigma_{s}(x) \mathrm{d} s\right| \leq(n+1)^{r} \quad \text { for } n \geq n_{0}(x),
$$

where $n_{0}(x) \geq 0$ is an integer that is finite for $\omega$-a.e. $x \in \mathfrak{X}$. In this case, we can write

$$
\left|\frac{1}{t} \int_{0}^{t} \sigma_{s}(x) \mathrm{d} s-\frac{1}{n} \int_{0}^{n} \sigma_{s}(x) \mathrm{d} s\right| \leq \frac{1}{n}\left|\int_{n}^{n+\hat{t}} \sigma_{s}(x) \mathrm{d} s\right|+\frac{1}{n^{2}}\left|\int_{0}^{n} \sigma_{s}(x) \mathrm{d} s\right| .
$$

where $n$ is the integer part of $t$ and $\hat{t}=t-n$. It follows from (60) that the first term on the right-hand side goes to zero for a.e. $x \in \mathfrak{X}$, and the second goes to zero in view of (59). Combining this with (59), we obtain (19). Thus, it remains to establish (60).

Let us fix an arbitrary $r \in(0,1)$ and denote by $\xi_{n}(x)$ the expression on the left-hand side of (60). In view of the first relation in (11), we have

$$
\xi_{n}(x)=\sup _{0 \leq t \leq 1}\left|\int_{n}^{n+t}\left(\mathrm{e}^{s \mathcal{L}} x, \varsigma \mathrm{e}^{s \mathcal{L}} x\right) \mathrm{d} s\right|=\sup _{0 \leq t \leq 1}\left|\left(x, \varsigma_{n, t} x\right)\right|, \quad \varsigma_{n, t}:=\int_{n}^{n+t} \varsigma_{s} \mathrm{~d} s .
$$

Suppose we have constructed a sequence $\left\{B_{n}\right\}$ of selfadjoint elements of $\mathcal{T}$ such that, for any $n \geq 0$,

$$
\sup _{0 \leq t \leq 1}\left|\left(x, \varsigma_{n, t} x\right)\right| \leq\left(x, B_{n} x\right), \quad\left\|B_{n}\right\|_{1} \leq C,
$$

where $C>0$ does not depend on $n$. In this case, introducing the events $A_{n}=\left\{x \in \mathfrak{X} \mid \xi_{n}(x) \geq(n+1)^{r}\right\}$, for sufficiently small $\varepsilon>0$, we can write

$$
\omega\left(A_{n}\right) \leq \mathrm{e}^{-\varepsilon(n+1)^{r}} \omega\left(\mathrm{e}^{\varepsilon \xi_{n}}\right) \leq \mathrm{e}^{-\varepsilon(n+1)^{r}}\left(\operatorname{det}\left(I-2 \varepsilon D B_{n}\right)\right)^{-1 / 2},
$$

where we used the fact that the Gaussian measures on $\mathfrak{X}$ with covariance operators $D_{\varepsilon}^{\prime}=\left(D^{-1}-2 \varepsilon B_{n}\right)^{-1}$ and $D$ are equivalent, with the corresponding density given by (see (5))

$$
\Delta_{D_{\varepsilon}^{\prime} \mid D}(x)=\left(\operatorname{det}\left(I-2 \varepsilon D B_{n}\right)\right)^{1 / 2} \mathrm{e}^{\varepsilon\left(x, B_{n} x\right)} .
$$

In view of the second inequality in (61), the determinant in (62) is bounded from below by a positive number not depending on $n \geq 0$ for sufficiently small $\varepsilon>0$. Thus, the series $\sum_{n} \omega\left(A_{n}\right)$ converges, and by the Borel-Cantelli lemma, inequality (60) holds with an almost surely finite integer $n_{0}(x)$.

We now prove (61). From Assumption (G2) we derive

$$
M \geq D_{t}=\mathrm{e}^{t \mathcal{L}} D \mathrm{e}^{t \mathcal{L}^{*}} \geq m \mathrm{e}^{t \mathcal{L}} \mathrm{e}^{t \mathcal{L}^{*}},
$$

so that the uniform bound

$$
\left\|\mathrm{e}^{t \mathcal{L}}\right\| \leq\left(\frac{M}{m}\right)^{1 / 2}
$$


holds. Since $\varsigma \in \mathcal{T}$ is selfadjoint, one has $|(x, \varsigma x)| \leq(x,|\varsigma| x)$ for all $x \in \mathcal{K}$. Hence

$$
\sup _{0 \leq t \leq 1}\left|\left(x, \varsigma_{n, t} x\right)\right| \leq \int_{n}^{n+1}\left|\left(\mathrm{e}^{s \mathcal{L}} x, \varsigma \mathrm{e}^{s \mathcal{L}} x\right)\right| \mathrm{d} s \leq \int_{n}^{n+1}\left(\mathrm{e}^{s \mathcal{L}} x,|\varsigma| \mathrm{e}^{s \mathcal{L}} x\right) \mathrm{d} s=\left(x, B_{n} x\right),
$$

where

$$
B_{n}=\int_{n}^{n+1} \mathrm{e}^{s \mathcal{L}^{*}}|\varsigma| \mathrm{e}^{s \mathcal{L}} \mathrm{d} s
$$

is a self-adjoint element of $\mathcal{T}$ such that

$$
\left\|B_{n}\right\|_{1} \leq \frac{M}{m}\|\varsigma\|_{1}
$$

The proof of Theorem 2.4 is complete.

\subsection{Proof of Theorem 2.5}

Part (1) Let $\left\{s_{n}\right\}$ be an arbitrary sequence converging to $\bar{\delta}$. Recall that $D^{-1}+\alpha T_{s_{n}}>0$ for $\alpha \in J_{s_{n}}$. Multiplying this inequality by $\mathrm{e}^{s_{n} \mathcal{L} / 2}$ from the right and by $\mathrm{e}^{s_{n} \mathcal{L}^{*} / 2}$ from the left, we obtain

$$
(1-\alpha) D_{-s_{n} / 2}^{-1}+\alpha D_{s_{n} / 2}^{-1}>0,
$$

for any $\alpha \in J_{s_{n}}$. Invoking Assumptions (G2)-(G3), we can pass to the limit in the last inequality to get

$$
(1-\alpha) D_{-}^{-1}+\alpha D_{+}^{-1} \geq 0
$$

for any $\alpha \in \bar{J}$. Taking $\alpha=1+\bar{\delta}$ and $\alpha=-\bar{\delta}$ and performing some simple estimation, we obtain inequality (20). Furthermore, it follows from (20) that $\alpha Q<1$ for $\alpha \in(-\bar{\delta}, 1+\bar{\delta})$, whence we conclude that the operator function (21) is analytic in the cut plane $\mathbb{C}_{+} \cup \mathbb{C}_{-} \cup(-\bar{\delta}, 1+\bar{\delta})$.

Part (2) We first prove the existence of the limit in (22). To this end, we shall apply Vitali's convergence theorem to the sequence of functions

$$
h_{n}(\alpha)=\frac{1}{t_{n}} e_{t_{n}}(\alpha), \quad n \geq 1, \quad \alpha \in J_{t_{n}} .
$$

By the very definition of $\hat{\delta}$, for any $\varepsilon>0$ there is $N_{\varepsilon}$ such that, for all $n \geq N_{\varepsilon}$, the function $h_{n}$ is analytic in the cut plane $\mathbb{C}_{-} \cup \mathbb{C}_{+} \cup \hat{J}_{\varepsilon}$ where

$$
\hat{J}_{\varepsilon}=(-\hat{\delta}+\varepsilon, 1+\hat{\delta}-\varepsilon) \subset J_{t_{n}} .
$$

By the proof of Part (4) of Theorem 2.4 (more precisely Eq. (58)), the functions $h_{n}$ are uniformly bounded in any disk or radius less than $\delta$ around $\alpha=0$. By the Cauchy estimate, the same is true of their derivatives $h_{n}^{\prime}$.

Let $K_{0}$ be the compact subset of $\left(\mathbb{C}_{-} \cup \mathbb{C}_{+} \cup \hat{J}_{\varepsilon}\right) \backslash\{0\}$ described on the left of Figure 1. From Definition (48) we infer

$$
D_{t_{n}}(\alpha)=D^{1 / 2}\left(1+\alpha Q_{n}\right)^{-1} D^{1 / 2}=z D^{1 / 2}\left(z-Q_{n}\right)^{-1} D^{1 / 2}, \quad z=-\frac{1}{\alpha},
$$

where $Q_{n}=D^{1 / 2} T_{t_{n}} D^{1 / 2}$ is a selfadjoint element of $\mathcal{T}$. By definition, $\alpha \in J_{t_{n}}$ iff $I+\alpha Q_{n}>0$, i.e.,

$$
\operatorname{sp}\left(Q_{n}\right) \subset\left(-\left(1+\delta_{t_{n}}\right)^{-1}, \delta_{t_{n}}^{-1}\right) \subset\left(-(1+\hat{\delta}-\varepsilon)^{-1},(\hat{\delta}-\varepsilon)^{-1}\right)
$$

for all $n \geq N_{\varepsilon}$. Since the function $\alpha \mapsto z=-1 / \alpha$ maps $K_{0}$ to a set which is uniformly separated from $\operatorname{sp}\left(Q_{n}\right)$ (see Figure 1), it follows from the spectral theorem that

$$
\sup _{\substack{n \geq N_{\varepsilon} \\ \alpha \in K_{0}}}\left\|D_{t_{n}}(\alpha)\right\| \leq\|D\| \sup _{\substack{n \geq N_{\varepsilon} \\-z^{-1} \in K_{0}}} \frac{|z|}{\operatorname{dist}\left(z, \operatorname{sp}\left(Q_{n}\right)\right)}<\infty .
$$



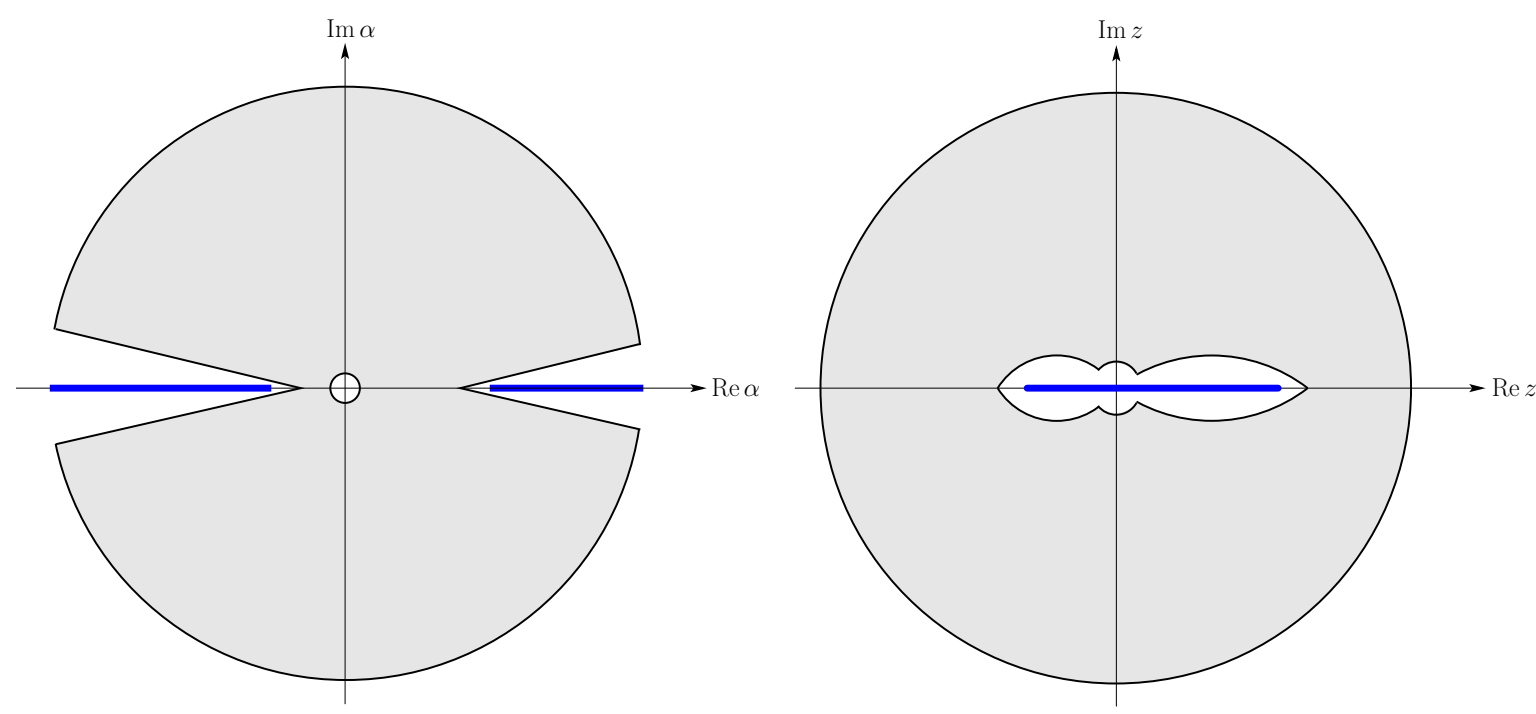

Figure 1: A compact region $K_{0} \subset\left(\mathbb{C}_{-} \cup \mathbb{C}_{+} \cup \hat{J}_{\varepsilon}\right) \backslash\{0\}$ and its image under the map $\alpha \mapsto z=-1 / \alpha$. The thick lines in the $\alpha$-plane are the cuts $\mathbb{R} \backslash \hat{J}_{\varepsilon}$. By Eq. (64), if $n \geq N_{\varepsilon}$, then the spectrum of $Q_{n}$ lies inside the thick line of the $z$-plane.

Applying Lemma 4.1 (3) to Eq. 46 (recall that the first term on the right hand side of the latter vanishes) and integrating Eq. (39) to express $T_{t_{n}}$ we obtain

$$
h_{n}^{\prime}(\alpha)=-\frac{1}{2 t_{n}} \operatorname{tr}\left(D_{t_{n}}(\alpha) T_{t_{n}}\right)=\int_{0}^{1} \operatorname{tr}\left(D_{t_{n}}(\alpha) \varsigma_{-s t_{n}}\right) \mathrm{d} s .
$$

The bound (63) further yields

$$
\left|h_{n}^{\prime}(\alpha)\right| \leq \frac{M}{m}\|\varsigma\|_{1}\left\|D_{t_{n}}(\alpha)\right\|,
$$

and the previous estimate allows us to conclude that the sequence $\left\{h_{n}^{\prime}\right\}_{n \geq N_{\varepsilon}}$ is uniformly bounded in $K_{0}$. Summing up, we have shown that $\left\{h_{n}^{\prime}\right\}_{n \geq N_{\varepsilon}}$ is uniformly bounded on any compact subset of $\mathbb{C}_{-} \cup \mathbb{C}_{+} \cup \hat{J}_{\varepsilon}$ and since $h_{n}(0)=0$, the same is true of the sequence $\left\{h_{n}\right\}_{n \geq N_{\varepsilon}}$. By Part (1) of Theorem 2.4, the sequence $\left\{h_{n}(\alpha)\right\}$ converges for $\alpha \in \underline{J}$. By Vitali's theorem (see Section I.A.12 in [GR]), we conclude that the sequence $\left\{h_{n}\right\}$ converges uniformly on any compact subset of $\mathbb{C}_{-} \cup \mathbb{C}_{+} \cup \hat{J}_{\varepsilon}$, and the limit is an analytic function on it. Since $\varepsilon>0$ was arbitrary, we see that the middle term in (22) is well defined for any $\alpha \in \mathbb{C}_{-} \cup \mathbb{C}_{+} \cup \hat{J}$ and is an analytic function on this domain.

To prove the second equality in (22), it suffices to establish it for $\alpha \in \underline{J}$, because both left- and right-hand sides are analytic functions on $\mathbb{C}_{-} \cup \mathbb{C}_{+} \cup \hat{J}$. The lower bound (57) shows that $D_{t}(\alpha)$ is bounded and strictly positive for all $t \in \mathbb{R}$ and $\alpha \in(-\delta, 1+\delta)$. It follows from Eq. (37) and Lemma 4.1 (1) that $\ell_{\omega_{t} \mid \omega} \in L^{1}\left(\mathfrak{X}, \mathrm{d} \omega_{D_{t}(\alpha)}\right)$. Moreover, Eq. (45) shows that for $f \in L^{1}\left(\mathfrak{X}, \mathrm{d} \omega_{D_{t}(\alpha)}\right)$

$$
\omega_{D_{t}(\alpha)}(f)=\frac{\omega\left(\mathrm{e}^{\alpha \ell_{\omega_{t} \mid \omega}} f\right)}{\omega\left(\mathrm{e}^{\alpha \ell_{\omega_{t} \mid \omega}}\right)} .
$$

Using this relation with $f=\ell_{\omega_{t} \mid \omega}$, integrating the identity

$$
\mathrm{e}^{\alpha \ell_{\omega_{t} \mid \omega}}=1+\int_{0}^{\alpha} \mathrm{e}^{\gamma \ell_{\omega_{t} \mid \omega}} \ell_{\omega_{t} \mid \omega} \mathrm{d} \gamma
$$

against $\omega$, and applying Fubini's theorem, we obtain

$$
\omega\left(\mathrm{e}^{\alpha \ell_{\omega_{t} \mid \omega}}\right)=1+\int_{0}^{\alpha} \omega\left(\mathrm{e}^{\gamma \ell_{\omega_{t} \mid \omega}}\right) \omega_{D_{t}(\gamma)}\left(\ell_{\omega_{t} \mid \omega}\right) \mathrm{d} \gamma .
$$


Resolving this integral equation (which reduces to a linear differential equation) for $\alpha \mapsto \omega\left(\mathrm{e}^{\alpha \ell_{\omega_{t} \mid \omega}}\right)$, we derive

$$
\omega\left(\mathrm{e}^{\alpha \ell_{\omega_{t} \mid \omega}}\right)=\exp \left(\int_{0}^{\alpha} \omega_{D_{t}(\gamma)}\left(\ell_{\omega_{t} \mid \omega}\right) \mathrm{d} \gamma\right) .
$$

Taking the logarithm, dividing by $t$, and using (41), we obtain

$$
\frac{1}{t} e_{t}(\alpha)=\frac{1}{t} \int_{0}^{\alpha} \omega_{D_{t}(\gamma)}\left(\ell_{\omega_{t} \mid \omega}\right) \mathrm{d} \gamma=\frac{1}{t} \int_{0}^{\alpha} \int_{0}^{t} \omega_{D_{t}(\gamma)}\left(\sigma_{-s}\right) \mathrm{d} s \mathrm{~d} \gamma=\int_{0}^{\alpha} \int_{0}^{1} \omega_{D_{t}(\gamma)}\left(\sigma_{-t s}\right) \mathrm{d} s \mathrm{~d} \gamma .
$$

It follows from (34) and the first relation in (11) that

$$
\omega_{D_{t}(\gamma)}\left(\sigma_{-t s}\right)=\operatorname{tr}\left(D_{t}(\gamma) \varsigma_{-t s}\right)=\operatorname{tr}\left(\mathrm{e}^{-t s \mathcal{L}} D_{t}(\gamma) \mathrm{e}^{-t s \mathcal{L}^{*}} \varsigma\right)=\operatorname{tr}\left(\left((1-\gamma) D_{-t s}^{-1}+\gamma D_{t(1-s)}^{-1}\right)^{-1} \varsigma\right) .
$$

Combining this with Hypothesis (G3) and a continuity property of the trace, we derive

$$
\lim _{t \rightarrow \infty} \omega_{D_{t}(\gamma)}\left(\sigma_{-t s}\right)=\operatorname{tr}\left(\bar{D}_{\gamma} \varsigma\right)=\omega_{\bar{D}_{\gamma}}(\sigma) \text { for } \gamma \in(-\delta, 1+\delta), s \in(0,1),
$$

where we set $\bar{D}_{\gamma}=\left((1-\gamma) D_{-}^{-1}+\gamma D_{+}^{-1}\right)^{-1}$. The bound (58) allows us to apply the dominated convergence theorem to Eq. (66), and conclude that

$$
e(\alpha)=\lim _{t \rightarrow \infty} \frac{1}{t} e_{t}(\alpha)=\int_{0}^{\alpha} \int_{0}^{1} \omega_{\bar{D}_{\gamma}}(\sigma) \mathrm{d} s \mathrm{~d} \gamma=\int_{0}^{\alpha} \operatorname{tr}\left(\bar{D}_{\gamma} \varsigma\right) \mathrm{d} \gamma, \quad \alpha \in(-\delta, 1+\delta) .
$$

Writing $\bar{D}_{\gamma}=D_{-}^{1 / 2}(I-\gamma Q)^{-1} D_{-}^{1 / 2}$, we further get

$$
e(\alpha)=\int_{0}^{\alpha} \operatorname{tr}\left(D_{-}^{1 / 2}(I-\gamma Q)^{-1} D_{-}^{1 / 2} \varsigma\right) \mathrm{d} \gamma
$$

and performing the integral yields Eq. (22) for $\alpha \in(-\delta, 1+\delta)$.

Finally, to prove (23), it suffices to note that if $\alpha$ does not belong to the closure of $\hat{J}$ then, for infinitely many $n \geq 1, \alpha \notin J_{t_{n}}$ and by Proposition $2.3(2), e_{t_{n}}(\alpha)=+\infty$.

Part (3) The required properties of the rate function $\hat{I}$ follow from (15) and elementary properties of the Legendre transform. Thus, we shall only prove (25). In doing so, we shall assume that the interval $\hat{J}$ is finite; in the opposite case, the result follows immediately from the Gärtner-Ellis theorem; see Section 4.5.3 in [DZ]. Moreover, we shall consider only the non-degenerate situation in which $\omega_{+}(\sigma)>0$. The analysis of the case $\omega_{+}(\sigma)=0$ is similar and easier.

Let us extend $\hat{e}(\alpha)$ to the endpoints of the interval $\hat{J}$ by the relation

$$
\hat{e}(\alpha)=\limsup _{t \rightarrow+\infty} \frac{1}{t} e_{t}(\alpha), \quad \alpha \in\{-\hat{\delta}, 1+\hat{\delta}\} .
$$

Since the extended function $\hat{e}$ is convex and, hence, continuous at any point where it is finite, the Legendre transform of $e(-\alpha)$ coincides with $\hat{I}$ defined by (24). In view of a well-known result on the large deviation upper bound (e.g., see Theorem 4.5.3 in [DZ]), the following inequality holds for any closed subset $F \subset \mathbb{R}$ :

$$
\limsup _{n \rightarrow \infty} \frac{1}{t_{n}} \log \omega\left(\left\{x \in \mathfrak{X} \mid \frac{1}{t_{n}} \int_{0}^{t_{n}} \sigma_{s}(x) \mathrm{d} s \in F\right\}\right) \leq-\inf _{s \in F} \hat{I}(s) .
$$

Since $\hat{I}$ is also continuous, this upper bound easily implies that (23) will be established if we prove the inequality

$$
\liminf _{n \rightarrow \infty} \frac{1}{t_{n}} \log \omega\left(\left\{x \in \mathfrak{X} \mid \frac{1}{t_{n}} \int_{0}^{t_{n}} \sigma_{s}(x) \mathrm{d} s \in O\right\}\right) \geq-\inf _{s \in O} \hat{I}(s),
$$

where $O \subset \mathbb{R}$ is an arbitrary open set. A standard argument shows that it suffices to prove (68) for any open interval $\mathcal{J} \subset \mathbb{R}$. Let us set

$$
s^{-}=-\lim _{\alpha \uparrow 1+\hat{\delta}} \hat{e}^{\prime}(\alpha), \quad s^{+}=-\lim _{\alpha \downarrow-\hat{\delta}} \hat{e}^{\prime}(\alpha) .
$$


In view of the local version of the Gärtner-Ellis theorem (see Theorem 4.65 in $^{6}$ [JOPP]), relation (25) is true for any interval $\mathcal{J} \subset\left(s^{-}, s^{+}\right)$. Thus, it suffices to consider the case when $\mathcal{J}=\mathcal{J}_{s, \varepsilon}=(s-\varepsilon, s+\varepsilon)$, where $\pm\left(s-s_{ \pm}\right) \geq 0$. The proof of (68) is divided into several steps.

Step 1: Reduction. We first show that the required inequality will be established if we prove that, for any $\hat{s} \in \mathbb{R}$ satisfying the inequality $\pm\left(\hat{s}-s_{ \pm}\right) \geq 0$ and any $\varepsilon>0$,

$$
\liminf _{n \rightarrow \infty} \frac{1}{t_{n}} \log \omega\left(B_{n}(\hat{s}, \varepsilon)\right) \geq-\hat{I}(\hat{s} \pm \varepsilon)
$$

where $B_{n}(\hat{s}, \varepsilon)=\left\{x \in \mathfrak{X}|| t_{n}^{-1} \ell_{\omega_{t_{n}} \mid \omega}+\hat{s} \mid<\varepsilon\right\}$. Indeed, we have

$$
\hat{I}(s)=\left\{\begin{array}{cc}
-(1+\hat{\delta}) s-e^{-} & \text {for } s \leq s^{-}, \\
\hat{\delta} s-e^{+} & \text {for } s \geq s^{+},
\end{array}\right.
$$

where $e^{-}$(respectively, $e^{+}$) is the limit of $\hat{e}(\alpha)$ as $\alpha \uparrow 1+\hat{\delta}$ (respectively, $\alpha \downarrow-\hat{\delta}$ ). In particular, the rate function $\hat{I}$ is everywhere finite and continuous. It follows from (69) and inequality (68) with $\mathcal{J} \subset\left(s^{-}, s^{+}\right)$ that

$$
\begin{aligned}
\lim _{\varepsilon \rightarrow 0^{+}} \liminf _{n \rightarrow \infty} \frac{1}{t_{n}} \log \omega\left(\left\{x \in \mathfrak{X} \mid \frac{1}{t_{n}} \int_{0}^{t_{n}} \sigma_{s}(x) \mathrm{d} s \in \mathcal{J}_{\hat{s}, \varepsilon}\right\}\right) & =\lim _{\varepsilon \rightarrow 0^{+}} \liminf _{n \rightarrow \infty} \frac{1}{t_{n}} \log \omega\left(B_{n}(\hat{s}, \varepsilon)\right) \\
& \geq-\hat{I}(\hat{s}),
\end{aligned}
$$

where $\hat{s} \in \mathbb{R}$ is any point. A well-known (and simple) argument implies the required lower bound (68) for any interval $\mathcal{J} \subset \mathbb{R}$. Thus, we need to establish (69). To simplify the notation, we shall consider only the case when $\hat{s} \geq s_{+}$(assuming that $s_{+}<\infty$ ).

Step 2: Shifted measures. Let us fix $\hat{s} \geq s^{+}$and denote $\tilde{e}_{t}(\alpha)=e_{t}(-\alpha)$ and $\tilde{e}(\alpha)=\hat{e}(-\alpha)$. Since $\tilde{e}_{t_{n}}^{\prime}$ is a monotone increasing function mapping the interval $-J_{t_{n}}=\left(-1-\delta_{t_{n}}, \delta_{t_{n}}\right)$ onto $(-\infty, \infty)$ (see (46)), for any $n \geq 1$ there is a unique number $\alpha_{n} \in-J_{t_{n}}$ such that $\tilde{e}_{t_{n}}^{\prime}\left(\alpha_{n}\right)=t_{n} \hat{s}$. Following a well-known idea in the theory of large deviations, let us define a sequence of measures $\nu_{n}$ on $\mathfrak{X}$ by their densities

$$
\Delta_{\nu_{n} \mid \omega}=\exp \left(-\alpha_{n} \ell_{\omega_{t_{n}} \mid \omega}-\tilde{e}_{t_{n}}\left(\alpha_{n}\right)\right) \text {. }
$$

Suppose we have proved that

$$
\liminf _{n \rightarrow \infty} \nu_{n}\left(B_{n}(\hat{s}, \varepsilon)\right)>0 .
$$

In this case, assuming that $\alpha_{n}>0$, we can write

$$
\begin{aligned}
\omega\left(B_{n}(\hat{s}, \varepsilon)\right) & =\int_{B_{n}(\hat{s}, \varepsilon)} \exp \left(\alpha_{n} \ell_{\omega_{t_{n}} \mid \omega}+\tilde{e}_{t_{n}}\left(\alpha_{n}\right)\right) \mathrm{d} \nu_{n} \\
& \geq \exp \left(t_{n} \alpha_{n}(-\hat{s}-\varepsilon)+\tilde{e}_{t_{n}}\left(\alpha_{n}\right)\right) \nu_{n}\left(B_{n}(\hat{s}, \varepsilon)\right),
\end{aligned}
$$

whence it follows that

$$
\liminf _{n \rightarrow \infty} \frac{1}{t_{n}} \log \omega\left(B_{n}(\hat{s}, \varepsilon)\right) \geq \liminf _{n \rightarrow \infty}\left(\alpha_{n}(-\hat{s}-\varepsilon)+\frac{1}{t_{n}} \tilde{e}_{t_{n}}\left(\alpha_{n}\right)\right) .
$$

If we know that

$$
\lim _{n \rightarrow \infty} \alpha_{n}=\hat{\delta}, \quad \liminf _{n \rightarrow \infty} \frac{1}{t_{n}} \tilde{e}_{t_{n}}\left(\alpha_{n}\right) \geq e^{+},
$$

then $\alpha_{n}>0$ for $n$ large enough and inequality (72) and relation (70) immediately imply the required result (69). Thus, we need to prove (71) and (73).

\footnotetext{
${ }^{6}$ In the formulation of Theorem 4.65 in [JOPP], it is required that the limit of $t_{n}^{-1} e_{t_{n}}(\alpha)$ as $n \rightarrow \infty$ should exist for any $\alpha$ in the closure of $\hat{J}$. However, the same proof works also in the case when the limits exist only for $\alpha \in \hat{J}$.
} 
Step 3: Proof of (73). Since $\alpha_{n} \in-J_{t_{n}}$ and $\delta_{t_{n}} \rightarrow \hat{\delta}$, the first relation in (73) will be established if we show that

$$
\liminf _{n \rightarrow \infty} \alpha_{n}=\hat{\delta} .
$$

Suppose this is not the case. Then there is $\varepsilon>0$ and a sequence $n_{k} \rightarrow+\infty$ such that $-1 \leq \alpha_{n_{k}} \leq \hat{\delta}-\varepsilon$, where the first inequality follows from the fact that $\tilde{e}_{t_{n}}^{\prime}\left(\alpha_{n}\right) \geq 0$ and $\tilde{e}_{t_{n}}^{\prime}(-1) \leq 0$. To simplify notation, we assume that the entire sequence $\left\{\alpha_{n}\right\}$ satisfies this inequality. It follows that

$$
s^{+} \leq \hat{s}=\frac{1}{t_{n}} \tilde{e}_{t_{n}}^{\prime}\left(\alpha_{n}\right) \leq \frac{1}{t_{n}} \tilde{e}_{t_{n}}^{\prime}(\hat{\delta}-\varepsilon) \text { for any } n \geq 1 .
$$

Since $\frac{1}{t_{n}} e_{t_{n}}(\alpha)$ are convex functions converging to the smooth function $\tilde{e}(\alpha)$ for $\alpha \in-\hat{J}$, by Theorem 25.7 in [Rock], we have

$$
\lim _{n \rightarrow \infty} \frac{1}{t_{n}} \tilde{e}_{t_{n}}^{\prime}(\alpha)=\tilde{e}^{\prime}(\alpha) \quad \text { for any } \alpha \in-\hat{J},
$$

and the limit is uniform on any compact subset of $-\hat{J}$. Comparing this with (75), we see that $s^{+} \leq \tilde{e}^{\prime}(\hat{\delta}-\varepsilon)$. It follows that $\tilde{e}^{\prime}$ is constant on the interval $[\hat{\delta}-\varepsilon, \hat{\delta}]$ and, hence, by analyticity and the first relation in (15), the function $e(\alpha)$ vanishes. This contradicts the assumption that $\omega_{+}(\sigma)>0$ and proves (74).

We now establish the second relation in (73). For any $\gamma \in(0, \hat{\delta})$, we have

$$
\tilde{e}_{t_{n}}\left(\alpha_{n}\right)=\tilde{e}_{t_{n}}(\gamma)+\int_{\gamma}^{\alpha_{n}} \tilde{e}_{t_{n}}^{\prime}(\alpha) \mathrm{d} \alpha \geq \tilde{e}_{t_{n}}(\gamma)+\left(\alpha_{n}-\gamma\right) \tilde{e}_{t_{n}}^{\prime}(0),
$$

where we used the facts that $\tilde{e}^{\prime}$ is nondecreasing and that $\alpha_{n}>\gamma$ for sufficiently large $n \geq 1$, in view of the first relation in (73). It follows that

$$
\liminf _{n \rightarrow \infty} \frac{1}{t_{n}} \tilde{e}_{t_{n}}\left(\alpha_{n}\right) \geq \tilde{e}(\gamma)+(\hat{\delta}-\gamma) \tilde{e}^{\prime}(0) .
$$

Passing to the limit as $\gamma \rightarrow \hat{\delta}$, we obtain the required inequality.

Step 4: Proof of (71). Let us introduce trace class operators

$$
Q_{n}=D^{1 / 2} T_{t_{n}} D^{1 / 2}, \quad M_{n}=t_{n}^{-1}\left(I-\alpha_{n} Q_{n}\right)^{-1} Q_{n}, \quad n \geq 1 .
$$

Since $\alpha_{n} \in-J_{t_{n}}$, the operator $I-\alpha_{n} Q_{n}$ is strictly positive and, hence, invertible, so that $M_{n}$ is well defined. Suppose we have shown that

$$
\nu_{n}\left(f\left(X_{n}\right)\right)=\mu\left(f\left(Y_{n}\right)\right), \quad X_{n}=-t_{n}^{-1} \ell_{\omega_{t_{n}} \mid \omega}, \quad Y_{n}=\frac{1}{2}\left(x, M_{n} x\right), \quad n \geq 1,
$$

where $f: \mathbb{R} \rightarrow \mathbb{R}$ is an arbitrary bounded measurable function and $\mu$ is the centered Gaussian measure on $\mathfrak{X}$ with the covariance operator $I$. In this case, taking $f$ to be the indicator function of the interval $\mathcal{J}_{\hat{s}, \varepsilon}$, we can write

$$
\nu_{n}\left(B_{n}(\hat{s}, \varepsilon)\right)=\mu\left(\left\{x \in \mathfrak{X}|| Y_{n}(x)-\hat{s} \mid<\varepsilon\right\}\right)=: p_{n}(\varepsilon) \quad \text { for any } n \geq 1 .
$$

Thus, the required assertion will be established if we prove that

$$
\inf _{n \geq 1} p_{n}(\varepsilon)>0 \quad \text { for any } \varepsilon>0 .
$$

To this end, let us assume that we have proved that

$$
\mathfrak{M}:=\sup _{n \geq 1}\left\|M_{n}\right\|_{1}<\infty, \quad \operatorname{tr}\left(M_{n}\right)=2 \hat{s} .
$$

We now use the following lemma, whose proof is given in the end of this subsection (cf. Lemma 2 in [BD, Section 3].) 
Lemma 4.2 Let $\mu$ be the centered Gaussian measure on $\mathfrak{X}$ with the covariance operator I. Then for any positive numbers $\kappa$ and $\varepsilon$ there is $p(\kappa, \varepsilon)>0$ such that

$$
\mu(\{x \in \mathfrak{X}||(x, M x)-\operatorname{tr}(M) \mid<\varepsilon\}) \geq p(\kappa, \varepsilon)
$$

for any selfadjoint operator $M \in \mathcal{T}$ satisfying the inequality $\|M\|_{1} \leq \kappa$.

In view of (78), we have

$$
Y_{n}(x)-\hat{s}=\left(x, \frac{1}{2} M_{n} x\right)-\operatorname{tr}\left(\frac{1}{2} M_{n}\right) .
$$

Applying Lemma 4.2 with $\kappa=2 \mathfrak{M}$, we see that (77) holds. Thus, to complete the proof of the theorem, it remains to establish (76) and (78).

Step 5: Proof of the auxiliary assertions. Simple approximation and analyticity arguments show that, to prove (76), is suffices to consider the case in which $f(x)=\mathrm{e}^{\gamma x}$, where $\gamma \in \mathbb{R}$ is sufficiently small. Thus, we need to check that

$$
\nu_{n}\left(\exp \left(-\gamma t_{n}^{-1} \ell_{\omega_{t_{n}} \mid \omega}\right)\right)=\mu\left(\mathrm{e}^{\gamma Y_{n}}\right) .
$$

Recalling the construction of $\alpha_{n}$ and using the relation $\tilde{e}_{t}(\alpha)=-\frac{1}{2} \log \operatorname{det}\left(I-\alpha Q_{t}\right)$ (see (46)), we write

$$
\begin{aligned}
\nu_{n}\left(\exp \left(-\gamma t_{n}^{-1} \ell_{\omega_{t_{n}} \mid \omega}\right)\right) & =\int_{\mathfrak{X}} \exp \left(-\left(\gamma t_{n}^{-1}+\alpha_{n}\right) \ell_{\omega_{t_{n}} \mid \omega}-\tilde{e}_{t_{n}}\left(\alpha_{n}\right)\right) \omega(\mathrm{d} x) \\
& =\exp \left(\tilde{e}_{t_{n}}\left(\gamma t_{n}^{-1}+\alpha_{n}\right)-\tilde{e}_{t_{n}}\left(\alpha_{n}\right)\right)=\operatorname{det}\left(I-\gamma M_{n}\right)^{-1 / 2} .
\end{aligned}
$$

This expression coincides with the right-hand side of (80).

Finally, to prove (78), we first note that the equality follows immediately from the choice of $\alpha_{n}$ and the relation $\tilde{e}_{t}^{\prime}(\alpha)=\frac{1}{2} \operatorname{tr}\left(\left(I-\alpha Q_{t}\right)^{-1} Q_{t}\right)$. To establish the inequality, we start by using (39) and (63) to get the bound

$$
\left\|Q_{n}\right\|_{1} \leq \int_{0}^{t_{n}}\left\|D^{1 / 2} \varsigma_{-s} D^{1 / 2}\right\|_{1} \mathrm{~d} s \leq \frac{M^{2}}{m} t_{n}\|\varsigma\|_{1} .
$$

Writing the spectral decomposition of the compact self-adjoint operator $M_{n}$, we easily show that

$$
M_{n}^{-}=t_{n}^{-1}\left(I+\alpha_{n} Q_{n}^{-}\right)^{-1} Q_{n}^{-},
$$

where $A^{+}$and $A^{-}$stand the positive and negative parts of a selfadjoint operator $A$, and we used that fact that $\alpha_{n}>0$ for sufficiently large $n$ (see (74)). Combining this relation with (81), we derive

$$
\operatorname{tr}\left(M_{n}^{-}\right)=t_{n}^{-1} \operatorname{tr}\left(\left(I+\alpha_{n} Q_{n}^{-}\right)^{-1} Q_{n}^{-}\right) \leq \frac{M^{2}}{m}\|\varsigma\|_{1} .
$$

Recalling the second relation in (78), we conclude that

$$
\left\|M_{n}\right\|_{1}=\operatorname{tr}\left(\left|M_{n}\right|\right)=\operatorname{tr}\left(M_{n}+2 M_{n}^{-}\right) \leq 2\left(\hat{s}+\frac{M^{2}}{m}\|\varsigma\|_{1}\right) .
$$

The proof of Theorem 2.5 is complete.

Proof of Lemma 4.2. We set $Y(x)=(x, M x)$ and note that $\mu(Y)=\operatorname{tr}(M)$. Let us denote by $\left\{P_{I}, I \subset \mathbb{R}\right\}$ the family of spectral projections for $M$ and, given a number $\theta>0$, write $M=M^{\leq \theta}+M^{>\theta}$, where $M^{\leq \theta}=M P_{[-\theta, \theta]}$. Accordingly, we represent $Y$ in the form

$$
Y(x)=Y^{\leq \theta}(x)+Y^{>\theta}(x), \quad Y^{\leq \theta}(x)=\left(x, M^{\leq \theta} x\right)-\operatorname{tr}\left(M^{\leq \theta}\right) .
$$

Now note that the random variables $Y^{\leq \theta}$ and $Y^{>\theta}$ are independent under the law $\mu$. It follows that the probability $P(M, \varepsilon)$ given by the left-hand side of (79) satisfies the inequality

$$
P(M, \varepsilon) \geq \mu\left(\left\{\left|Y^{>\theta}\right|<\varepsilon / 2,\left|Y^{\leq \theta}\right|<\varepsilon / 2\right\}\right)=\mu\left(\left\{\left|Y^{>\theta}\right|<\varepsilon / 2\right\}\right) \mu\left(\left\{\left|Y^{\leq \theta}\right|<\varepsilon / 2\right\}\right) .
$$


We claim that both factors on the right-hand side of this inequality are separated from zero. Indeed, to estimate the first factor, we note that

$$
\kappa \geq\|M\|_{1} \geq \theta \operatorname{rank}\left(M^{>\theta}\right),
$$

where $\operatorname{rank}\left(M^{>\theta}\right)=: N_{\theta}$ stands for the rank of $M^{>\theta}$. Denoting by $\lambda_{j}$ the eigenvalues of $M$ indexed in the non-increasing order of their absolute values, we see that

$$
\left.\mid Y^{>\theta}(x)\right)|=| \sum_{j=1}^{N_{\theta}} \lambda_{j}\left(x_{j}^{2}-1\right)\left|\leq \kappa \sum_{j=1}^{N_{\theta}}\right| x_{j}^{2}-1 \mid,
$$

where $\left\{x_{j}\right\}$ are the coordinates of $x$ in the orthonormal basis formed of the eigenvectors of $M$. Combining this with (83), we derive

$$
\left.\mu\left\{\mid Y^{>\theta}(x)\right) \mid<\varepsilon / 2\right\} \geq \mu\left\{\sum_{j=1}^{N_{\theta}}\left|x_{j}^{2}-1\right|<\frac{\varepsilon}{2 \kappa}\right\} \geq \prod_{j=1}^{N_{\theta}} \mu\left\{\left|x_{j}^{2}-1\right|<\left(2 \kappa N_{\theta}\right)^{-1} \varepsilon\right\} \geq p(\delta)^{\kappa / \theta},
$$

where $\delta=\varepsilon \theta /\left(2 \kappa^{2}\right)$, and $p(\delta)>0$ is the probability of the event $\left|x^{2}-1\right|<\delta$ under the one-dimensional standard normal law. To estimate the second factor in (82), we use the Chebyshev inequality:

$$
\begin{aligned}
\mu\left\{\left|Y^{\leq \theta}(x)\right|<\varepsilon / 2\right\} & =1-\mu\left\{Y^{\leq \theta}(x) \geq \varepsilon / 2\right\}-\mu\left\{-Y^{\leq \theta}(x) \geq \varepsilon / 2\right\} \\
& \geq 1-\mu\left(\exp \left(\gamma Y^{\leq \theta}-\gamma \varepsilon / 2\right)\right)+\mu\left(\exp \left(-\gamma Y^{\leq \theta}-\gamma \varepsilon / 2\right)\right),
\end{aligned}
$$

where $\gamma>0$ is sufficiently small and will be chosen later. We have

$$
\begin{aligned}
\mu\left(\exp \left(\gamma Y^{\leq \theta}\right)\right) & =\exp \left\{-\gamma \operatorname{tr}\left(M^{\leq \theta}\right)-\frac{1}{2} \log \operatorname{det}\left(I-\gamma M^{\leq \theta}\right)\right\} \\
& =\exp \left\{-\frac{1}{2} \operatorname{tr}\left(2 \gamma M^{\leq \theta}+\log \left(I-2 \gamma M^{\leq \theta}\right)\right)\right\} .
\end{aligned}
$$

Now note that if $4|\gamma| \theta \leq 1$, then

$$
2 \gamma M^{\leq \theta}+\log \left(I-2 \gamma M^{\leq \theta}\right)=\sum_{n=2}^{\infty} \frac{\left(-2 \gamma M^{\leq \theta}\right)^{n}}{n} .
$$

Recalling that $\|M \leq \theta\| \leq \theta$ and $\left\|M^{\leq \theta}\right\|_{1} \leq \kappa$ and using the inequality $|\operatorname{tr}(A B)| \leq\|A\|_{1}\|B\|$, it follows that

$$
|\operatorname{tr}(2 \gamma M \leq \theta+\log (I-2 \gamma M \leq \theta))| \leq \sum_{n=2}^{\infty}|2 \gamma \theta|^{n-1} 2|\gamma| \kappa \leq 8 \kappa \gamma^{2} \theta
$$

Substituting this into (85), we see that, if $|\gamma| \leq(4 \theta)^{-1}$, then $\mu\left(\exp \left(\gamma Y^{\leq \theta}\right)\right) \leq \exp \left(4 \kappa \gamma^{2} \theta\right)$. A similar estimate holds for $\mu\left(\exp \left(-\gamma Y^{\leq \theta}\right)\right)$. Combining these inequalities with (84) and choosing $\gamma=\frac{\varepsilon}{16 \kappa \theta}$, we derive

$$
\mu\left\{\left|Y^{\leq \theta}(x)\right|<\varepsilon / 2\right\} \geq 1-2 \exp \left(4 \kappa \gamma^{2} \theta-\gamma \varepsilon / 2\right)=1-2 \exp \left(-\frac{\varepsilon^{2}}{64 \kappa \theta}\right) .
$$

The right-hand side of this inequality can be made greater than zero by choosing a sufficiently small $\theta>0$ which will depend only on $\kappa$ and $\varepsilon$.

\subsection{Proof of Theorem 2.6}

The proof of this result is verty similar to that of Theorems 2.4 and 2.5, and we shall only outline the proof.

Part (1) Follows from Hölder's inequality as in the proof of Proposition 2.3 (2).

Part (2) Since $0 \in J_{t}^{+}$, the fact that $J_{t}^{+}$is an interval follows immediately from the following property: if $\alpha \in J_{t}^{+}$, then $\theta \alpha \in J_{t}^{+}$for $\theta \in(0,1)$. To prove the analyticity, note that, by Eq. (45), one has

$$
\mathrm{e}^{-\alpha \ell_{\omega_{t} \mid \omega}} \mathrm{d} \omega_{+}=\sqrt{\frac{\left(\operatorname{det}\left(I+D T_{t}\right)\right)^{-\alpha}}{\operatorname{det}\left(I-\alpha D_{+} T_{t}\right)}} \mathrm{d} \omega_{\left(D_{+}^{-1}-\alpha T_{t}\right)^{-1}} .
$$


This relation implies that the function

$$
\begin{aligned}
e_{t+}(\alpha) & =-\frac{\alpha}{2} \log \operatorname{det}\left(I+D_{+} T_{t}\right)-\frac{1}{2} \log \operatorname{det}\left(I-\alpha D_{+} T_{t}\right) \\
& =-\frac{\alpha}{2} \log \operatorname{det}\left(I+D^{1 / 2} T_{t} D^{1 / 2}\right)-\frac{1}{2} \log \operatorname{det}\left(I-\alpha D_{+}^{1 / 2} T_{t} D_{+}^{1 / 2}\right)
\end{aligned}
$$

is real analytic in $\alpha$ on the open interval defined by the condition $I-\alpha D_{+}^{1 / 2} T_{t} D_{+}^{1 / 2}>0$ and takes the value $+\infty$ on its complement (where the intersection of the spectrum of $I-\alpha D_{+}^{1 / 2} T_{t} D_{+}^{1 / 2}$ with the negative half-line is nonempty). The above inequality coincides with the one defining $J_{t}^{+}$.

Part (3) The fact that $\underline{J}^{+}$is an interval follows immediately from its definition. To prove that $J_{t}^{+} \supset(-\delta, \delta)$, note that, in view of Hypothesis (G2), for any $t, \alpha \in \mathbb{R}$ we have

$$
I-\alpha D_{+}^{1 / 2} T_{t} D_{+}^{1 / 2}=D_{+}^{1 / 2}\left(D_{+}^{-1}-\alpha\left(D_{t}^{-1}-D^{-1}\right)\right) D_{+}^{1 / 2} \geq \frac{\delta-|\alpha|}{\delta+1} .
$$

This expression is positive for $|\alpha|<\delta$.

To prove the existence of limit (27) and its analyticity on $\underline{J}^{+}$, we repeat the argument used in the proof of Theorem 2.5 (2). Namely, let us introduce the family of operators $D_{t}^{+}(\alpha)=\left(D_{+}^{-1}-\alpha T_{t}\right)^{-1}$, which are well defined for $\alpha \in(-\delta, \delta)$. Then the following analogue of relation (65) is valid:

$$
\omega_{D_{t}^{+}(\alpha)}(f)=\frac{\omega\left(\mathrm{e}^{-\alpha \ell_{t} \mid \omega} f\right)}{\omega\left(\mathrm{e}^{-\alpha \ell_{\omega_{t} \mid \omega}}\right)} \quad \text { for } f \in L^{1}\left(\mathfrak{X}, \mathrm{d} \omega_{D_{t}^{+}(\alpha)}\right) .
$$

The argument used in the derivation of (66) gives that

$$
\frac{1}{t} e_{t+}(\alpha)=-\int_{0}^{\alpha} \int_{0}^{1} \omega_{D_{t}^{+}(\gamma)}\left(\sigma_{-t s}\right) \mathrm{d} s \mathrm{~d} \gamma
$$

while Hypothesis (G2) and the relation $\mathrm{e}^{r \mathcal{L}} D_{+} \mathrm{e}^{r \mathcal{L}^{*}}=D_{+}$valid for $r \in \mathbb{R}$ imply that

$$
\mathrm{e}^{-t s \mathcal{L}} D_{t}^{+}(\gamma) \mathrm{e}^{-t s \mathcal{L}^{*}}=\left(D_{+}^{-1}-\gamma\left(D_{t(1-s)}^{-1}-D_{-t s}^{-1}\right)\right)^{-1} \leq M\left(1-\frac{|\gamma|}{\delta}\right)^{-1}
$$

Following again the argument in the proof of Theorem $2.5(2)$, for $\alpha \in(-\delta, \delta)$ we derive

$$
e_{+}(\alpha)=\lim _{t \rightarrow \infty} \frac{1}{t} e_{t+}(\alpha)=-\int_{0}^{\alpha} \omega_{\bar{D}_{1-\gamma}}(\sigma) \mathrm{d} \gamma
$$

Now note that $\bar{D}_{1-\gamma}=\vartheta \bar{D}_{\gamma} \vartheta$, whence it follows $\omega_{\bar{D}_{1-\gamma}}(\sigma)=\omega_{\bar{D}_{\gamma}}(\sigma \circ \vartheta)=-\omega_{\bar{D}_{\gamma}}(\sigma)$. Substituting this into (87) and recalling (67), we see that

$$
e_{+}(\alpha)=\int_{0}^{\alpha} \omega_{\bar{D}_{\gamma}}(\sigma) \mathrm{d} \gamma=e(\alpha) \text { for } \alpha \in(-\delta, \delta)
$$

We have thus established the existence of limit (27) on the interval $(-\delta, \delta) \subset \underline{J}^{+}$. The fact that it exists for any $\alpha \in \underline{J}^{+}$and defines a real-analytic function can be proved with the help of Vitali's theorem (cf. proof of Part (2) of Theorem 2.5). Finally, relation (28) is established by the same argument as (23).

Parts (4-6) The proofs of the large deviation principle, central limit theorem, and strong law of large numbers for the time average of the entropy production functional under the limiting law $\omega_{+}$are exactly the same as for $\omega$ (see Parts (3-5) of Theorem 2.4), and therefore we will omit them.

Parts (7) The fact that the functions $e_{+}(\alpha)$ and $e(\alpha)$ coincides on the intersection $\underline{J}^{+} \cap \underline{J}$ follows from (88) and their analyticity. The equality of the corresponding rate functions on a small interval around $\omega_{+}(\sigma)$ is a straightforward consequence of (88) and the definition of the Legendre transform. 


\section{References}

[BaCo] Baule, A., and Cohen E.G.D.: Steady state work fluctuations of a dragged particle under external and thermal noise. Phys. Rev. E 80, 011110 (2009).

[BGGZ] Bonetto, F., Gallavotti, G., Guiliani, A., and Zamponi, F.: Chaotic hypothesis, fluctuation theorem and singularities. J. Stat. Phys. 123, 39-54 (2006).

[BFL] Bercu, B., Gamboa, F., and Lavielle, M.: Sharp large deviations for Gaussian quadratic forms with applications. ESAIM Probab. Statist. 4, 1-24 (2000).

[BFR] Bercu, B., Gamboa, F., and Rouault, A.: Large deviations for quadratic forms of stationary Gaussian processes. Stoch. Proc. Appl. 71, 75-90 (1997).

[Bill] Billingsley, P.: Convergence of Probability Measures. John Wiley \& Sons, New York, 1999.

[BJMS] Baiesi, M., Jacobs, T., Maes, C., and Skantzos, S.: Fluctuation symmetries for work and heat. Phys. Rev. E 74, 021111 (2006).

[Br] Bryc, W.: A remark on the connection between the large deviation principle and the central limit theorem. Stat. Prob. Lett. 18, 253-256 (1993).

[BD] Bryc, W., and Dembo, A.: Large deviations for quadratic functionals of Gaussian processes. J. Theoret. Probab. 10, 307-332 (1997).

[CFS] Cornfeld, I.P., Fomin, S.V., and Sinai, Ya G.: Ergodic Theory. Springer, Berlin, 1982.

[DZ] Dembo, A., and Zeitouni, O.: Large Deviations Techniques and Applications. Springer, New York, 1998.

[ECM] Evans, D.J., Cohen, E.G.D., and Morriss, G.P.: Probability of second law violation in shearing steady flows. Phys. Rev. Lett. 71, 2401-2404 (1993).

[El] Ellis, R.S.: Entropy, Large Deviations, and Statistical Mechanics. Springer, Berlin (1985). Reprinted in the series Classics of Mathematics (2006).

[ES] Evans, D.J., and Searles, D.J.: Equilibrium microstates which generate second law violating steady states. Phys Rev. E 50, 1645-1648 (1994).

[Fa] Farago, J.: Injected power fluctuations in Langevin equations. J. Stat. Phys. 107, 781-803 (2002).

[HP] Hille, E., and Phillips, R.S.: Functional Analysis and Semigroups. American Mathematical Society, Providence, Rhode Island, 1957.

[HRS] Harris, R.J., Rákos, A., and Schütz, G.M.: Breakdown of Gallavotti-Cohen symmetry for stochastic dynamics. Europhys. Lett. 75, 227 (2006)

[GC1] Gallavotti, G., and Cohen, E.G.D.: Dynamical ensembles in nonequilibrium statistical mechanics. Phys. Rev. Lett. 74, 2694-2697 (1995).

[GC2] Gallavotti, G., and Cohen, E.G.D.: Dynamical ensembles in stationary states. J. Stat. Phys. 80, 931-970 (1995).

[GK] Gohberg, I.C., and Krein, M.G.: Introduction to the Theory of Linear Nonselfadjoint Operators. American Mathematical Society, Providence, Rhode Island, 1969.

[GR] Gunning, R.C., and Rossi, H.: Analytic Functions of Several Complex Variables. Prentice-Hall, Englewood Cliffs, N.J., 1965. 
[JLP] Jakšić, V., Landon, B., and Pillet, C.-A.: Entropic fluctuations in XY chains and reflectionless Jacobi matrices. Annales Henri Poincaré 14, 1775-1800 (2013).

[JOPP] Jakšić, V., Ogata, Y., Pautrat, Y., and Pillet, C.-A.: Entropic fluctuations in quantum statistical mechanics-an introduction. In Quantum Theory from Small to Large Scales, J. Fröhlich, M. Salmhofer, V. Mastropietro, W. De Roeck and L.F. Cugliandolo editors. Oxford University Press, Oxford, 2012.

[JPR] Jakšić, V., Pillet, C.-A., and Rey-Bellet, L.: Entropic fluctuations in statistical mechanics I. Classical dynamical systems. Nonlinearity 24, 699-763 (2011).

[JPS] Jakšić, V., Pillet, C.-A., and Shirikyan, A.: Entropic fluctuations in thermally driven harmonic networks. In preparation (2015).

[Lan] Landon, B.: Stationary non-equilibrium states of infinite harmonic systems. Master's thesis, McGill University (2013).

[RM] Rondoni, L., and Mejìa-Monasterio, C.: Fluctuations in non-equlibrium statistical mechanics: models, mathematical theory, physical mechanisms. Nonlinearity 20, 1-37 (2007).

[Rock] Rockafellar, R.T.: Convex Analysis. Princeton University Press, Princeton, N.J., 1997.

[Rud] Rudin, W.: Real and Complex Analysis. McGraw-Hill, New York, 1987.

[Si] Simon, B.: Trace Ideals and their Applications. Second edition. Mathematical Surveys and Monograph, 120. American Mathematical Society, Providence, Rhode Island, 2005.

[Vi1] Visco, P., et all.: Fluctuations of power injection in randomly driven granular gases. J. Stat. Phys. 125, 533-568 (2006).

[Vi2] Visco, P.: Work fluctuation for a Brownian particle between two thermostats. J. Stat. Mech., P06006 (2006).

[ZC] van Zon, R., and Cohen, E.G.D.: An extension of the fluctuation theorem. Phys. Rev. Lett. 91, 110601 (2003). 